\title{
Optimized Strain Long-Period Fiber Grating (LPFG) Sensors Operating at the Dispersion Turning Point
}

\author{
Ignacio Del Villar, Omar Fuentes, Francesco Chiavaioli, Member, IEEE, Jesus M. Corres, Member, IEEE, \\ and Ignacio R. Matias, Senior Member, IEEE
}

\begin{abstract}
Two phenomena for enhancing the sensitivity of longperiod fiber gratings are combined toward an increase of the sensitivity to strain of this type of devices: the dispersion turning point (DTP) and the cladding diameter reduction by an etching process. The results prove that sensitivities up to $20 \mathrm{pm} / \mu \varepsilon$ can be attained, which is a ten-fold improvement compared to the previous works. The sensitivity in the grating region, which is subjected to etching, does not depend on the order of the cladding mode responsible for the attenuation bands generated in the transmission spectrum, but on the proximity to the DTP for each mode order. On the other hand, the sensitivity to strain of the global structure, including the region without etching, can be increased for lower order modes in a perceptible way if the length of the etched region is smaller compared to the fiber region under stress. The experimental results are supported with simulations based on coupled-mode theory and on FIMMWAVE, which allows understanding the phenomena involved during the sensing process.
\end{abstract}

Index Terms-Dispersion turning point, etching, long period fiber grating, strain sensor.

\section{INTRODUCTION}

$\mathbf{L}$ ONG period fiber gratings (LPFGs) consist typically of a periodic perturbation in the core of a single mode optical fiber, which allows a co-propagating coupling of light from the guided mode in the fiber core to several modes guided in the cladding [1]-[3]. This provides LPFGs with sensitivity to strain, temperature, bending and refractive index of the surrounding medium [3]. In order to guarantee that a co-propagating coupling to the cladding modes occurs, the period must be typically higher than $100 \mu \mathrm{m}$ (FBGs are generated with shorter periods and, hence, a counter-propagating coupling is generated).

During the last two decades, three main ways of enhancing the sensitivity of LPFGs to the surrounding refractive index (SRI)

Manuscript received November 7, 2017; revised January 2, 2018; accepted January 2, 2018. This work was supported in part by the Spanish Ministry of Economy and Competitiveness (TEC2016-78047-R - AEI/FEDER, UE), in part by the Government of Navarra PI044 NANOSEN, and in part by the Pinar del Río University. (Corresponding author: Ignacio Del Villar.)

I. Del Villar, J. M. Corres, and I. R. Matias are with the Institute of Smart Cities and the Electrical and Electronic Engineering Department, Public University of Navarre, Pamplona 31006, Spain (e-mail: ignacio.delvillar@unavarra.es; jmcorres@unavarra.es; natxo@unavarra.es).

O. Fuentes is with the Department of Telecommunications and Electronics, Pinar del Río University, Pinar del Río 20100, Cuba (e-mail: omarf@upr.edu.cu).

F. Chiavaioli is with the "Nello Carrara" Institute of Applied Physics, National Research Council of Italy, Firenze 50019, Italy (e-mail: f.chiavaioli @ ifac.cnr.it).

Color versions of one or more of the figures in this paper are available online at http://ieeexplore.ieee.org.

Digital Object Identifier 10.1109/JLT.2018.2790434 have been explored: the selection of an adequate period that allows the LPFG to operate at the dispersion turning point (DTP) [4], the deposition of a thin-film that allows the device operating in mode transition [5], [6] and the reduction of the cladding diameter [7], [8]. The combination of these three effects allows attaining sensitivities comparable or even higher than surface plasmon resonance sensors (SPRs) [9], [10], which positions LPFGs as one of the most promising platforms in the field of optical sensors.

A high sensitivity to SRI is a good indicator for the ability of the sensor to be used as a chemical or a biological sensor, because the parameter to detect (e.g., an antigen, antibody, or any chemical species) is deposited on the surface of the sensor and, consequently, affects the medium surrounding the optical fiber [11]. However, few research has been devoted to the optimization of the strain sensitivity, which not necessarily follows the same rules as the SRI sensitivity.

In this work, focus is centered on the combination of two phenomena: the DTP and the cladding etching. By combining both effects, it will be demonstrated that, contrary to what happens with the sensitivity to SRI [8], coupling to lower order cladding modes does not allow attaining a better sensitivity. However, for each cladding mode, the sensitivity is improved when the DTP is approaching.

In addition, another way of improving the sensitivity is applying stress in a longer region than the one that has been etched. The sensitivity to strain is increased for lower order modes (i.e., shorter diameter) compared to higher order modes, and this improvement is better if the length of region under stress is much longer than the region that has been etched.

In Section II the experimental setup and the methods used for fabricating and simulating the LPFGs are described. In Section III the experimental results are detailed. Finally, some conclusions are presented in Section IV.

\section{EXPERIMENTAL SECTION}

LPFGs with a grating period of $191 \mu \mathrm{m}$ and a length of $19 \mathrm{~mm}$ were written in a photosensitive boron-germanium codoped optical fiber (Fibercore PS1250/1500) having mode field diameter of $9.6 \mu \mathrm{m}$, numerical aperture of 0.13 and cladding diameter of $125 \mu \mathrm{m}$.

The LPFGs were fabricated with the point-by-point inscription technique by using an excimer $\mathrm{KrF}$ laser source (LAMBDA Physik COMPex 110, Coherent Inc.) operating at a wavelength of $248 \mathrm{~nm}$, and working at constant pulse energy. The other 


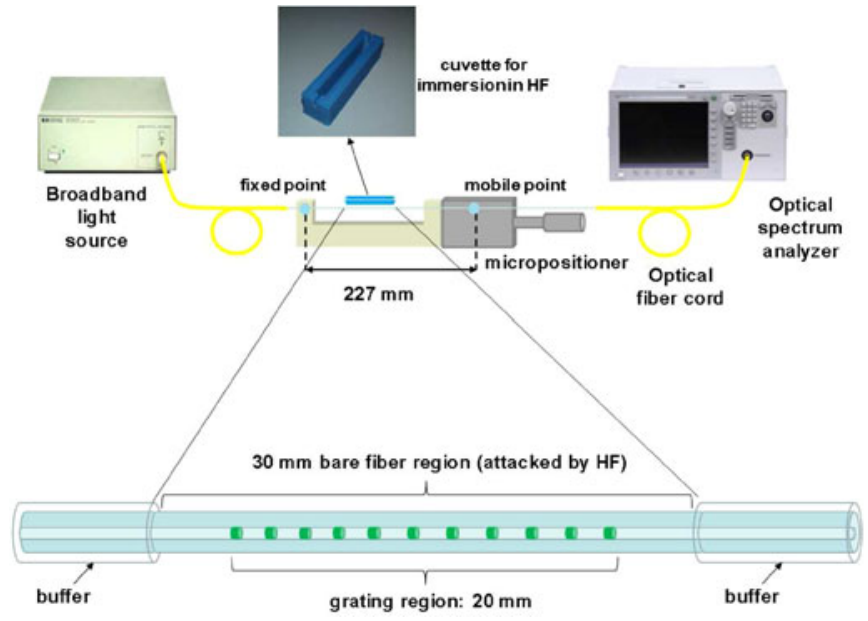

Fig. 1. Experimental setup used to etch the optical fiber containing an LPFG.

manufacturing parameters are as follows: energy of $140 \mathrm{~mJ}$ fluence of 200-300 mJ/ $\mathrm{cm}^{-2}$, and a repetition rate of $50 \mathrm{~Hz}$.

The laser beam was focused by using a cylindrical lens before being passed through a micrometric slit placed in front of the optical fiber, which determined the grating period. The fiber was kept straight during both the fabrication and the characterization processes to avoid any bending artifact.

The LPFGs were subjected to an etching process depicted in Fig. 1. The process was monitored with a transmission configuration setup. Light from an Agilent 83437A broadband source was launched into the LPFG during the etching process and the output light was monitored in an Agilent 86140B optical spectrum analyzer, which allowed observing the evolution of the attenuation bands and hence stopping at the adequate position.

Regarding the etching process, the fiber was introduced in a plastic cuvette filled with $40 \%$ hydrofluoric acid. The cuvette contains two 0.9-mm-wide grooves where the fiber segment to etch can be placed without affecting the rest of the fiber and without releasing acid out of the cuvette. The length of the fiber attacked by the acid was $30 \mathrm{~mm}$, enough to include the $19 \mathrm{~mm}$ long grating portion. In order to guarantee an adequate etching, the fiber was also fixed to an outer plastic holder and to a mobile point in a micropositioner, which ensured that the fiber was kept straight, avoiding bend-induced distortion of the transmission spectrum. The region not immersed in the cuvette was $197 \mathrm{~mm}$ long, for a global length of the fiber under stress of $227 \mathrm{~mm}$.

When the attenuation bands were positioned at a specific wavelength, the fiber was extracted from the cuvette and washed using water immediately after to eliminate the effects of acid.

The process can be repeated as many times as necessary in order to further reduce the cladding diameter, which allows obtaining attenuation bands corresponding with other cladding modes [8]. Moreover, if it is necessary to control the position of the attenuation bands with more accuracy, the LPFG can also be immersed in a more diluted HF solution.

After the etching process, it was essential to wait at least one hour to ensure that the LPFG was completely dry. Once dried, the LPFG was ready for performing strain measurements. To this purpose, as indicated in Fig. 1, the LPFG was manually stretched in steps of $0.1 \mathrm{~mm}$ (the transmission spectrum of the sensor was continuously monitored during the stretching process).

It is important to highlight that the diameter of the fiber was just reduced in the region of the LPFG immersed in the cuvette. Consequently, the strain in the grating can be obtained according to this expression:

$$
\varepsilon_{1}=\frac{\frac{\Delta L}{A_{1}}}{\left(\frac{L_{1}}{A_{1}}+\frac{L_{2}}{A_{2}}\right)}
$$

where $\Delta \mathrm{L}$ is the overall length increase of the LPFG region under stress, $L_{1}$ and $L_{2}$ are the lengths of the etched and nonetched fiber portions respectively, $A_{1}$ is the cross section of the etched fiber portion and $\mathrm{A}_{2}$ is the cross section of the non-etched fiber portion.

\section{THEORY}

The structures studied in this work were analyzed with two software tools: FIMMWAVE and a method based on coupledmode theory [13]. FIMMWAVE, which is a fast and efficient software for analyzing optical waveguides, was used for generating the transmission spectra, whereas the coupled-mode theory was used for obtaining the resonance wavelengths that meet the phase-matching condition (this last operation is less computationally demanding and cannot be directly obtained with FIMMWAVE).

Regarding FIMMWAVE, the propagation was calculated with FIMMPROP, a module integrated with FIMMWAVE. For LPFG sections, the finite difference method (FDM) was used, because it is the most accurate method available for a cylindrical waveguide.

The grating used in the simulations consisted of a square wave that emulates the point-by-point technique used during the inscription of the grating. The peak-to-peak modulation was $8 \times$ $10^{-4}$. Modes $\mathrm{LP}_{0,1}$ up to $\mathrm{LP}_{0,12}$ were analyzed for the period of the LPFGs used experimentally. $\mathrm{LP}_{0,11}$ was the higher-order cladding mode to which the core mode is coupled when the cladding diameter is $125 \mu \mathrm{m}$, i.e., before the fiber was etched.

By fitting the experimental results presented in Section IV with the theoretical ones, a core diameter of $6.9 \mu \mathrm{m}$ and a numerical aperture of 0.1313 at wavelength of $1300 \mathrm{~nm}$ was calculated, very close to 0.13 numerical aperture given by the manufacturer. For a better accuracy, a negative dispersion of $1.1 \times 10^{-7}(\lambda-1300)^{2}$ was added, where $\lambda$ is the operating wavelength.

It is well known that the resonance wavelength of an LPFG is determined by the phase-matching condition [1]-[4]:

$$
\lambda=\left[n_{\text {core }}(\lambda)-n_{\text {clad }}^{i}(\lambda)\right] \Lambda
$$

where $n_{\text {core }}(\lambda)$ is the effective refractive index of the propagating core mode at wavelength $\lambda, n_{\text {clad }}^{i}(\lambda)$ is effective the refractive index of the $i$ th cladding mode and $\Lambda$ is the period of the grating.

However, the modified phase-matching condition is [12]:

$$
\lambda=\left[n_{\text {core }}(\lambda)+\frac{s_{0}}{k_{0}} \varsigma_{\text {core }}-\left(n_{\text {clad }}^{i}(\lambda)+\frac{s_{0}}{k_{0}} \varsigma_{\text {clad }}^{i}\right)\right] \Lambda
$$


where the additional variables $s_{0}, k_{0}, \zeta_{\text {core }}$ and $\zeta_{\text {clad }}^{i}$ are the first Fourier component of the grating profile, the free space wavenumber, the self-coupling coefficient of the core mode and the self-coupling coefficient of the cladding mode, respectively, allows improving the accuracy of the equation towards a prediction of the position of the attenuation bands in the transmission spectrum [12]. Consequently, (3) will be used for the analysis performed in this section according to coupled-mode theory [13].

Using the chain rule of derivatives, the sensitivity to axial strain $\varepsilon$ can obtained from (3) as follows:

$$
\frac{d \lambda}{d \varepsilon}=\frac{d \lambda}{d m a t} \frac{d m a t}{d \varepsilon}+\Lambda \frac{d \lambda}{d \Lambda}
$$

where the first element on the right side of the equation is related to the material contribution extracted from expression (3):

$$
\text { mat }=\left[n_{\text {core }}(\lambda)+\frac{s_{0}}{k_{0}} \zeta_{\text {core }}-\left(n_{\text {clad }}^{i}(\lambda)+\frac{s_{0}}{k_{0}} \varsigma_{\text {clad }}^{i}\right)\right]
$$

This contribution results from the elasto-optic effect (i.e., the change in refractive index in both the fiber core and the cladding due to strain) and the Poisson's effect (i.e., the change in the transverse dimensions). The second element in expression (4) is the waveguide contribution, which depends on the slope $\mathrm{d} \lambda / \mathrm{d} \Lambda$ of the characteristic curve of the resonance band [3], [14].

The elasto-optic coefficient of silica is a well know parameter, -0.22 [4], [15], whereas the elasto-optic coefficient of the fiber core is not given in the literature because it depends on the doping level of the materials used by the manufacturer. The value that best fitted the experimental results in Section IV was -0.222 , which is in the range of the values explored in [4].

In order to analyze the effect of both the material and waveguide contributions in the optical fiber used in this work, two situations were taken into account: an LPFG without etching and an etched LPFG.

Fig. 2(a) shows the dependence of the coupling wavelength for cladding modes $\mathrm{LP}_{0,2}-\mathrm{LP}_{0,12}$ upon the period of the LPFGs used. For $\mathrm{LP}_{0,11}$ the DTP was observable [4]. This phenomenon is related to a high sensitivity. Consequently, the best option was to choose a period of $191 \mu \mathrm{m}$, which cuts the curve of $\mathrm{LP}_{0,11}$ cladding mode at two points very close to the DTP, where the sensitivity is very high.

It was also proved experimentally that, by reducing the cladding diameter of the optical fiber, coupling to lower order cladding modes in the DTP was possible [8]. This idea is confirmed in Fig. 2(b), where for an LPFG of cladding diameter $65.45 \mu \mathrm{m}$ a coupling to $\mathrm{LP}_{0,6}$ cladding mode at DTP is obtained.

In Fig. 3 the waveguide and the material contribution were analyzed with a focus on grating periods close to $191 \mu \mathrm{m}$, the period selected for the LPFGs analyzed in Section IV. To this purpose, the coupling wavelength for cladding mode $\mathrm{LP}_{0,11}$ was calculated in two conditions: one where the refractive index of both the fiber core and cladding was that corresponding to no strain applied to the LPFG, and another where the refractive index of both the fiber core and cladding was that corresponding to $3000 \mu \varepsilon$ applied to the LPFG. The effect is more evident near the DTP. However, it is very small if compared with the

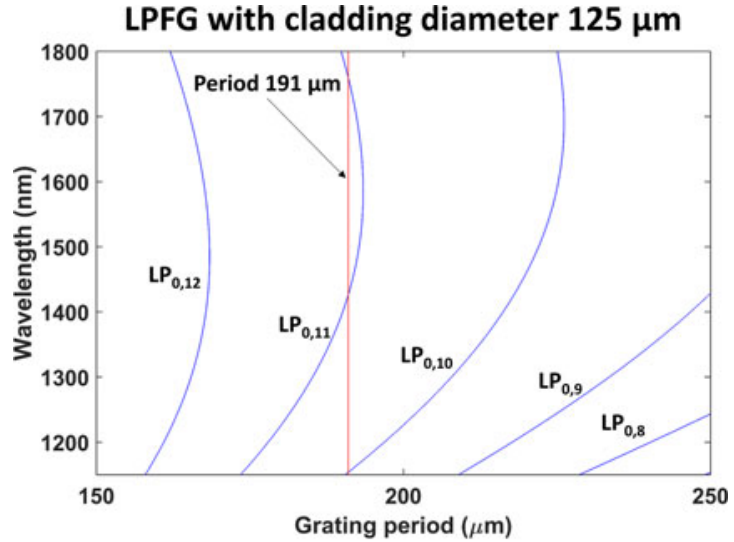

(a)

LPFG with cladding diameter $65.45 \mu \mathrm{m}$

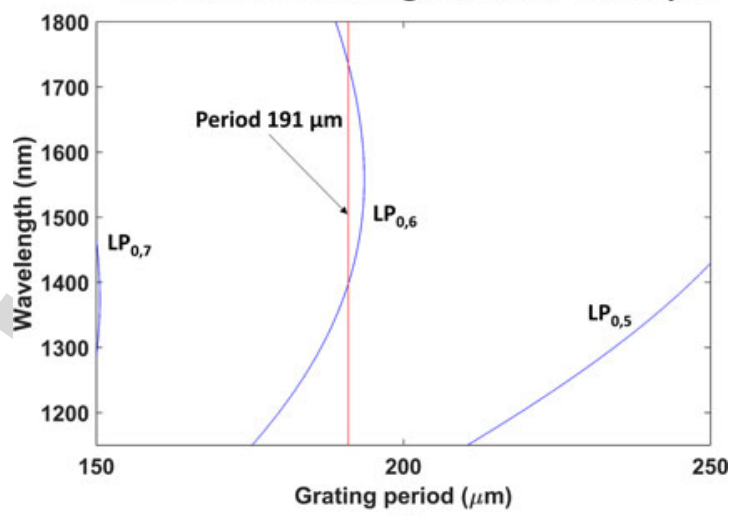

(b)

Fig. 2. Calculated variation of resonance wavelength with grating period: (a) For a cladding diameter of $125 \mu \mathrm{m}$ (for a period of $191 \mu \mathrm{m}$, the $\mathrm{LP}_{0,11}$ phase matching curve is intersected at two wavelengths very close to the dispersion turning point); (b) For a cladding diameter of $65.45 \mu \mathrm{m}$ (for a period of $191 \mu \mathrm{m}$, the $\mathrm{LP}_{0,6}$ phase matching curve is intersected at two wavelengths very close to the dispersion turning point).

effect of increasing the grating period by $0.3 \%$, equivalent to 219 $3000 \mu \varepsilon$ in the LPFG (see the vertical lines of $191 \mu \mathrm{m}, 0 \mu \varepsilon, \quad 220$ and $191.576 \mu \mathrm{m}, 3000 \mu \varepsilon$ ). In Fig. 3(a), for an LPFG with 221 cladding diameter $125 \mu \mathrm{m}$, a wavelength shift of approximately 222 $10 \mathrm{~nm}$ was observed (waveguide contribution) compared to less 223 than $1 \mathrm{~nm}$ induced by the material contribution. In other words, 224 the effect of the waveguide is predominant over the effect of 225 the material. The same effect was observed for an LPFG with 226 diameter $65.45 \mu \mathrm{m}$ in Fig. 3(b).

Another interesting conclusion that was extracted from the results obtained with two different diameters and shown in Fig. 3 was that the shape of the phase matching curve did not change. This indicates that the optimized sensitivity to strain is achieved for any mode as long as it operates at or close to the DTP point. Consequently, the basic rule for optimizing the sensitivity to strain should be to approach the DTP point. In addition to this, it is easy to observe in Fig. 3 the non-linear wavelength shift of the coupling wavelength as a function of the grating period in the proximities of the DTP.

\section{EXPERIMENTAL RESULTS}

According to Sections II and III, different cladding diameters were explored (see Table I). The diameter was estimated by 


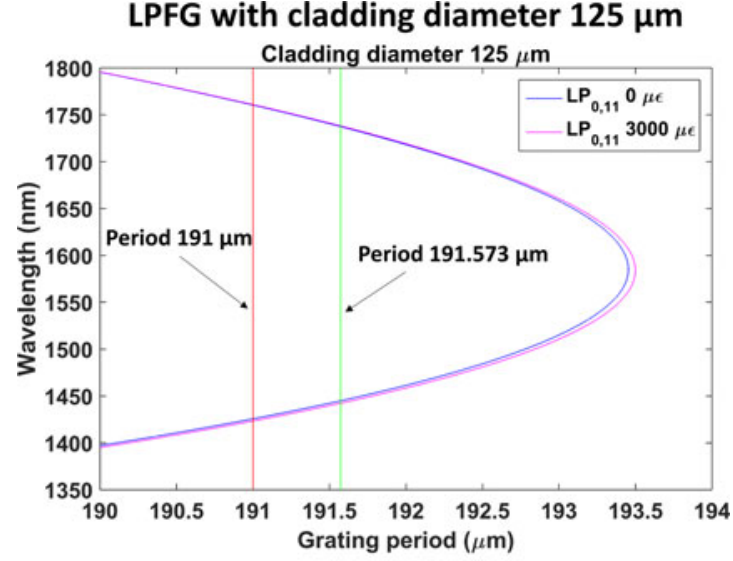

(a)

LPFG with cladding diameter $65.45 \mu \mathrm{m}$

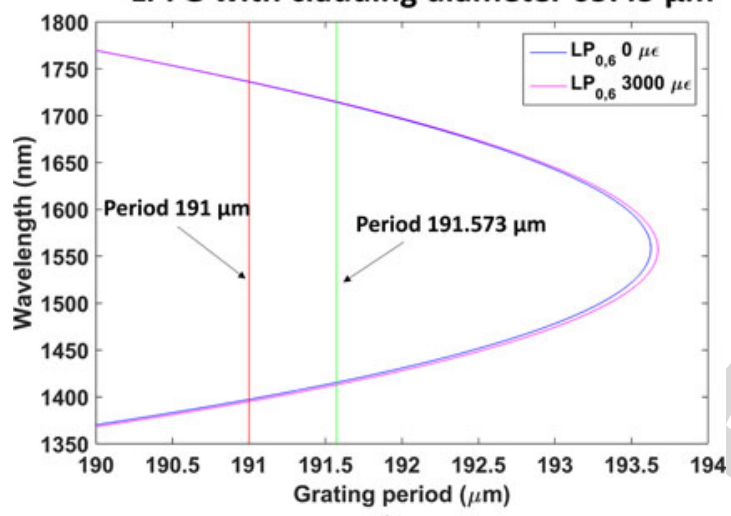

(b)

Fig. 3. Effect of the material in the variation of resonance wavelength with grating period ( 0 and $3000 \mu \varepsilon$ are compared), and effect of the waveguide (a period of $191 \mu \mathrm{m}$ is compared with $191.576 \mu \mathrm{m}$, which is a $0.3 \%$ more to represent the effect of $3000 \mu \varepsilon$ ): (a) For a cladding diameter of $125 \mu \mathrm{m}$. (b) For a cladding diameter of $65.45 \mu \mathrm{m}$.

TABLE I

LIST OF LPFG SENSORS OF PERIOD $191 \mu \mathrm{M}$

\begin{tabular}{lccl}
\hline Sensor & Mode order & Lambda $(\mathrm{nm})$ & Diameter $(\mu \mathrm{m})$ \\
\hline S0 & $\mathrm{LP}_{0,11}$ & 1461 & 125 \\
S1 & $\mathrm{LP}_{0,11}$ & 1489 & 124.68 \\
$\mathrm{~S} 2$ & $\mathrm{LP}_{0,11}$ & 1508 & 124.56 \\
$\mathrm{~S} 3$ & $\mathrm{LP}_{0,10}$ & 1317 & 115.82 \\
S4 & $\mathrm{LP}_{0,10}$ & 1393 & 113.98 \\
S5 & $\mathrm{LP}_{0,10}$ & 1456 & 113.10 \\
S6 & $\mathrm{LP}_{0,9}$ & 1392 & 101.94 \\
S7 & $\mathrm{LP}_{0,9}$ & 1455 & 101.16 \\
S8 & $\mathrm{LP}_{0,8}$ & 1390 & 89.90 \\
S9 & $\mathrm{LP}_{0,6}$ & 1462 & 65.31 \\
\hline
\end{tabular}

relating the position of the attenuation bands, after each etching process, with the same position obtained in the theoretical transmission spectra. It is important to note that a good correspondence between the estimation of the diameter and the experimental value of the diameter measured in a microscope was demonstrated in [16].

S0 was an LPFG without etching (diameter $125 \mu \mathrm{m}$ ). The device was subjected to stress according to the method explained in Section II. In Fig. 4(a), the separation between the two

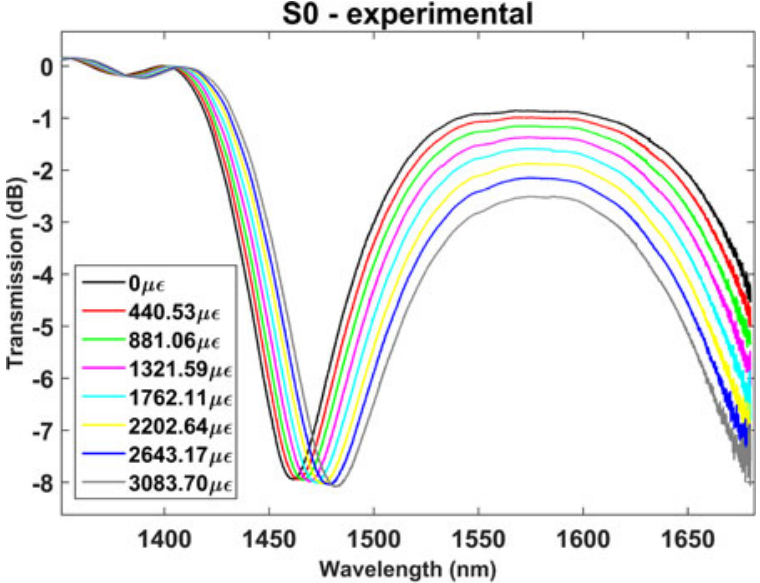

(a)

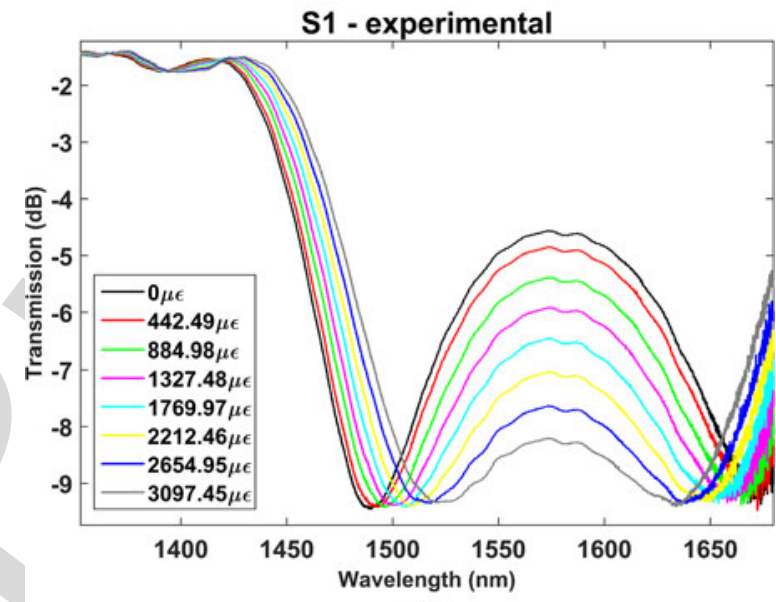

(b)

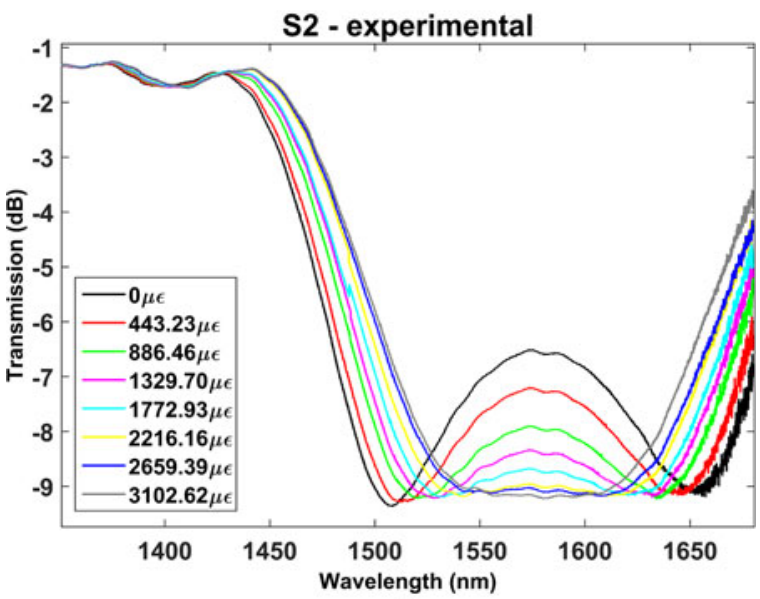

(c)

Fig. 4. Transmission spectra (experimental results) for: (a) S0; (b) S1; (c) S2.

attenuation bands observed in the optical spectrum is reduced 250 as a function of strain. This agrees with Fig. 3(a), where an 251 increase in the grating period leads to an approach to the DTP. 252

By performing a soft etching, we fabricated S1 with an esti- 253 mated diameter of $124.68 \mu \mathrm{m}$, according to the numerical results 254 presented in Fig. 5. Following the analysis in [8], [9], the sep- 255 aration of the attenuation bands decreases if the diameter of 256 an LPFG is reduced. This is what was observed in Fig. 4(b) if 257 


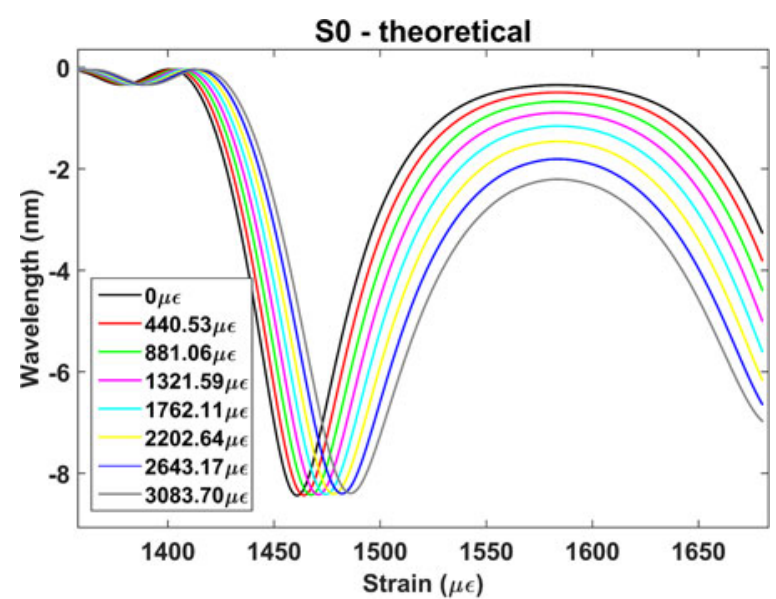

(a)

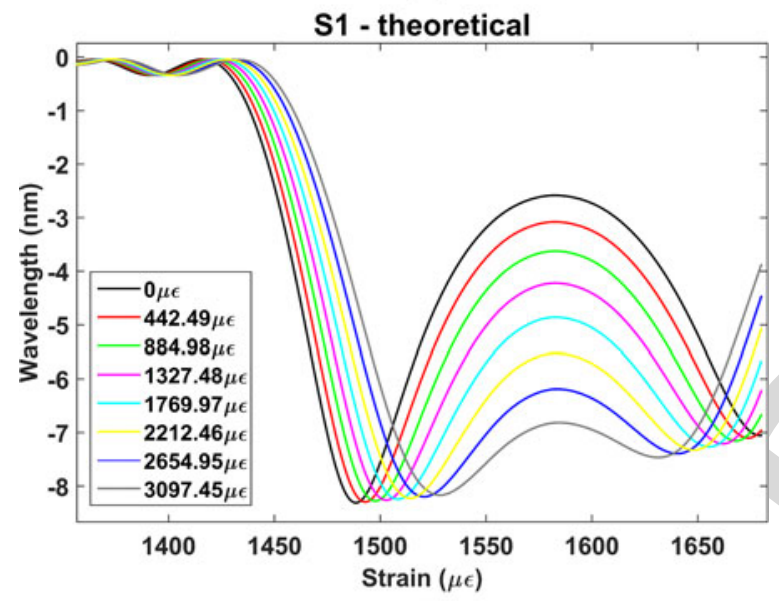

(b)

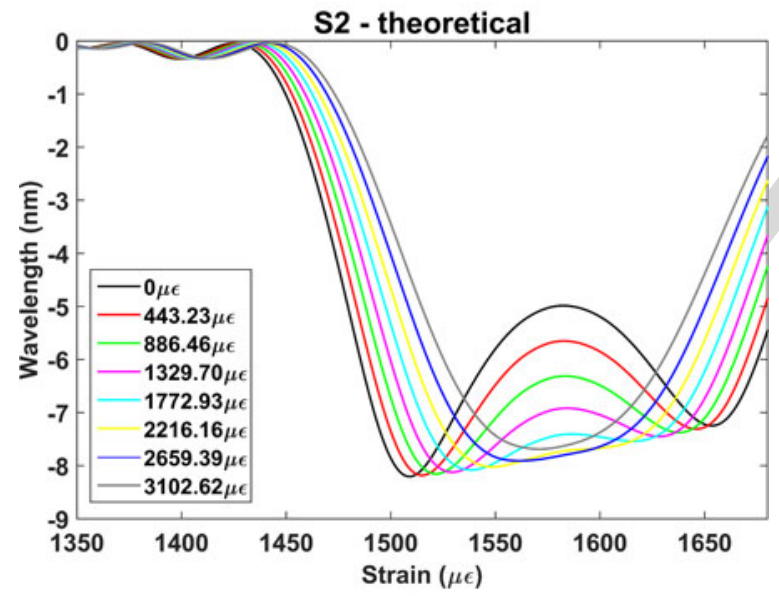

(c)

Fig. 5. Transmission spectra (theoretical results) for: (a) S0; (b) S1; (c) S2. compared with Fig. 4(a). The same occurred for sensor S2 in Fig. 4(c), with an estimated diameter of $124.56 \mu \mathrm{m}$. The theoretical results in Fig. 5 confirmed the experimental results of Fig. 4.

In addition, the central wavelength of the left band is plotted in Fig. 6 as a function of strain for all sensors analyzed in Figs. 4 and 5. The theoretical and experimental results allowed obtaining several conclusions. The sensitivity increases

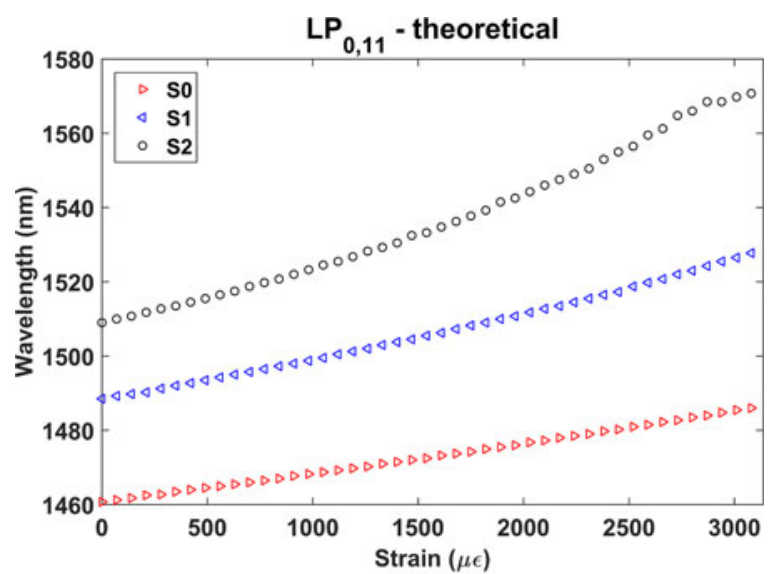

(a)

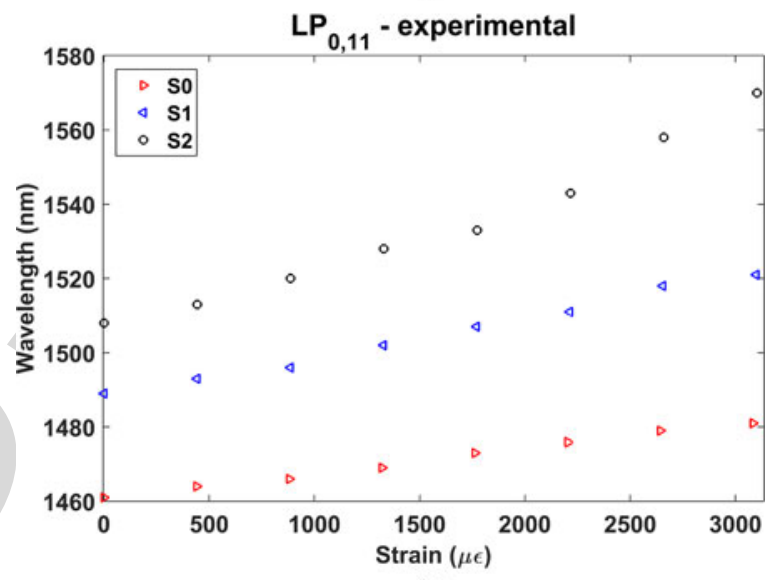

(b)

Fig. 6. Wavelength shift of $\mathrm{LP}_{0,11}$ left band as a function of strain for sensors S0, S1 and S2: (a) theoretical results; (b) experimental results.

if the diameter is reduced (the sensitivity of S0, S1 and S2 is 266 $6.5 \mathrm{pm} / \mu \varepsilon, 10.3 \mathrm{pm} / \mu \varepsilon$ and $20 \mathrm{pm} / \mu \varepsilon$, respectively, in the range 267 0-3100 $\mu \varepsilon)$. For the sake of comparison, the sensitivity in op- 268 timized LPFGs ranged from 0.5 to $2 \mathrm{pm} / \mu \varepsilon$ in [17], whereas 269 in [18] the maximum sensitivity was $2 \mathrm{pm} / \mu \varepsilon$. This indicates 270 that our best sensor improved the highest sensitivity attained in 271 these works by one order of magnitude.

A second conclusion is that if the separation between the attenuation bands is low, as S2 in Fig. 5, the relation between wavelength and strain is non-linear, whereas this relation is linear for sensor S0. Sensor S1 is in the middle between both situations. This agrees with what was observed in Fig. 3, where the phase matching curve is non-linear in the proximities of the DTP.

A harder etching was performed up to a diameter that allowed monitoring attenuation bands that were due to coupling to $\mathrm{LP}_{0,10}$ cladding mode. Three different diameters were analyzed: $115.82,113.98$ and $113.10 \mu \mathrm{m}$ (sensors S3, S4 and S5).

Theoretical and experimental data are presented in Fig. 7 for the central wavelength of the left band versus different values of strain. In all cases, the relation between strain and wavelength was linear. This can be explained because this time focused was centered on sensors working far from DTP: sensor S5 was working at the same wavelength as S0 in Fig. 6, where a 86

69




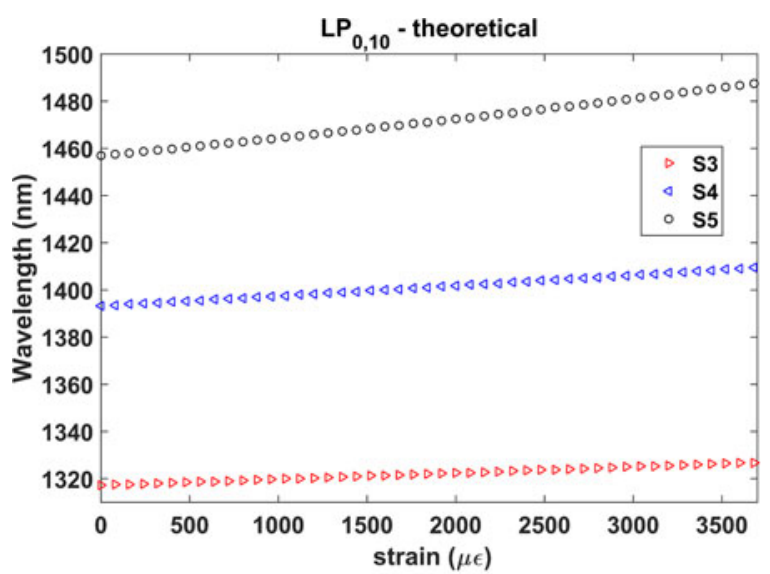

(a)

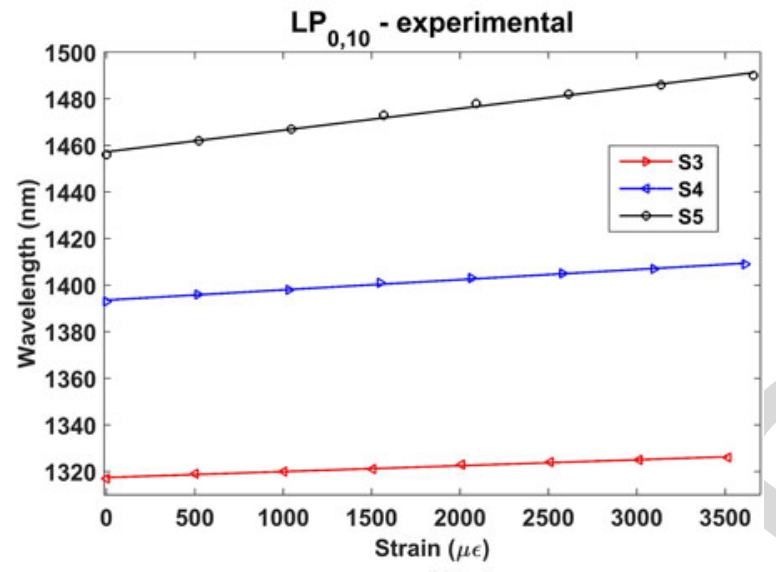

(b)

Fig. 7. Wavelength shift of $L P_{0,10}$ left band as a function of strain for sensors S3, S4 and S5: (a) theoretical results; (b) experimental results. linear performance was observed, and S3 and S4 were working at shorter wavelengths. In view of the linear shape of the plots, a Matlab linear regression model has been created (solid lines in Fig. 7) that fits with the experimental points. This model also allows obtaining the root-mean-square deviation along with the sensitivities for $\mathrm{S} 3, \mathrm{~S} 4$ and $\mathrm{S} 5$. The sensitivities are $2.5 \mathrm{pm} / \mu \varepsilon$, $4.4 \mathrm{pm} / \mu \varepsilon$ and $9.3 \mathrm{pm} / \mu \varepsilon$, respectively, in the range $0-3500 \mu \varepsilon$, whereas the root-mean-square deviations (RMSD) are respectively $0.348 \mathrm{~nm}, 0.432 \mathrm{~nm}$ and $1.05 \mathrm{~nm}$. The highest RMSD is obtained for S5, the sensor with the highest sensitivity and the sensor closer to DTP, where a non-linear dependence with strain is observed. In addition, as shown in Fig. 6, the sensitivity increases as the diameter is reduced.

In order to obtain more information on the influence of the mode order, other sensors with different diameters (S6, S7, S8 and S9) were analyzed. In Fig. 8, the performance of all sensors was compared. We divided them into two groups. Fig. 8(a) details the sensors working at a wavelength close to DTP. It seems that the sensitivity is similar. However, it is difficult to extract a general rule because the high sensitivity at this point is responsible for variations in the sensitivity of each sensor depending on small changes in the wavelength where it operates. On the other hand, Fig. 8(b) accounts for sensors operating far from the DTP, where the devices are not so sensitive to

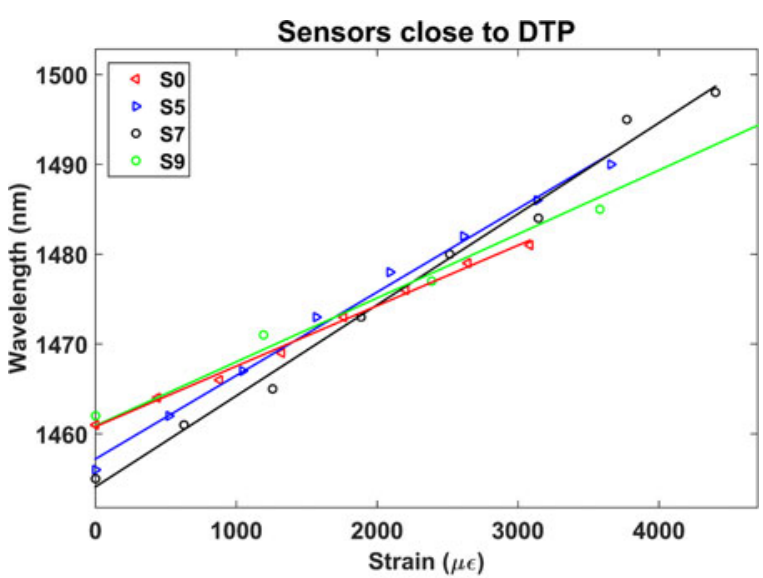

(a)

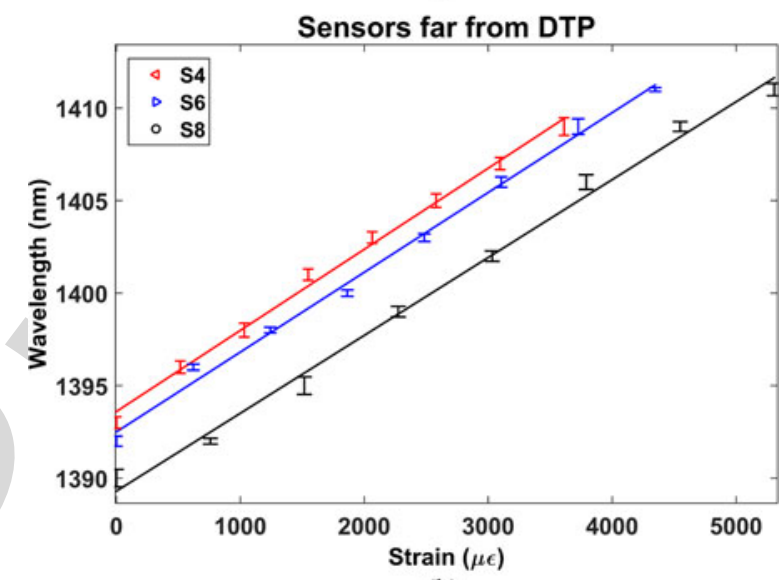

(b)

Fig. 8. Performance of LPFG sensors with different diameter: (a) S0, S5, S7 and S9 are close to DTP and the attenuation band is due to coupling to $\mathrm{LP}_{0,11}, \mathrm{LP}_{0,10}, \mathrm{LP}_{0,9}$ and $\mathrm{LP}_{0,6}$ respectively; (b) S4, S6 and $\mathrm{S} 8$ are far from DTP and the attenuation band is due to coupling to $\mathrm{LP}_{0,10}, \mathrm{LP}_{0,9}$ and $\mathrm{LP}_{0,8}$ respectively.

small variations in the operating wavelength and it is easier to 314 conclude that their sensitivity is quite similar. This indicates that 315 the mode order played no role on the sensitivity to strain of the 316 device. In other words, for each specific mode, the proximity to 317 the DTP determines the sensitivity of the device, whereas the 318 mode order has no influence on that. In this way, sensors can 319 be classified into two groups: operating at and far from DTP 320 point. Consequently, a soft etching to position the attenuation 321 bands in the optical spectrum is the best way for controlling the 322 sensitivity of the LPFG-based strain sensor.

It must be pointed out that the results presented in Figs. 4-8 324 represent the strain in the grating region of the LPFG. How- 325 ever, if the deformation in all the fiber structure is analyzed (see 326 Fig. 1), the sensitivity of the device is better for lower order 327 modes (i.e., lower diameter). Moreover, depending on the ra- 328 tio between the grating length and the complete LPFG region 329 length under stress, the sensitivity can be further improved. In 330 this sense, this ratio should be as low as possible. In the cases 331 analyzed in this work, if the strain on the global structure is con- 332 sidered, each $0.1 \mathrm{~mm}$ deformation in a structure of $227 \mathrm{~mm}$ is 333 $0.44 \mu \varepsilon$. Consequently, the $4 \mathrm{pm} / \mu \varepsilon$ sensitivity of S4, S6 and S8 334 in Fig. 8(b) becomes $5.19 \mathrm{pm} / \mu \varepsilon, 6.17 \mathrm{pm} / \mu \varepsilon$ and $6.82 \mathrm{pm} / \mu \varepsilon, \quad 335$ 
respectively, for the same sensors. These values are calculated by dividing the wavelength shift by $0.44 \mu \varepsilon$ multiplied by the seven $0.1 \mathrm{~mm}$ deformation steps analyzed in Fig. 8(b).

The RMSD of S4, S6 and S8 was also calculated: 0.432, 0.506 and $0.642 \mathrm{~nm}$ respectively. These values indicate no significant changes because the three sensors are positioned at a similar distance of the DTP. In addition, error bars representing the standard deviation in each point have been added for S4, S6 and S8 (the average standard deviation of the points in S4, S6 and S8 was $0.351,0.347$ and $0.332 \mathrm{~nm}$ respectively).

\section{CONCLUSION}

The sensitivity to strain of long period fiber gratings (LPFGs) operating close to the dispersion turning point (DTP) has been analyzed as a function of the cladding diameter.

The results obtained indicate that by accurately approaching the DTP it is possible to increase the sensitivity to strain of the device. Therefore, the sensitivity of previous works has been improved by a factor of 10 , attaining a sensitivity of $20 \mathrm{pm} / \mu \varepsilon$ in the best case.

On the other hand, unlike for LPFG-based refractometers, reducing the fiber diameter towards coupling to lower order cladding mode does not allow increasing the sensitivity of the etched region of the fiber. This indicates that a soft etching of the initial structure without etching towards a highest sensitivity is the best way of improving the performance of the device. However, if the etched region is small compared to the global region under stress, it is possible to improve the sensitivity of the global structure for lower order cladding modes, which opens the path towards the design of different combinations of length for both the etched and the non-etched regions. In this sense, the fabrication of short gratings is crucial towards the possibility to develop different designs.

The other well-known phenomenon that could be combined with the dispersion turning point and the cladding diameter reduction is the mode transition, which according to [9], [10] allows increasing the sensitivity to the surrounding refractive index changes of LPFGs exponentially. However, this requires the nanodeposition of a thin-film and, consequently, the number of variables to take into account increases in a great manner, which is beyond the scope of this work.

\section{REFERENCES}

[1] A. M. Vengsarkar, P. J. Lemaire, J. B. Judkins, V. Bhatia, T. Erdogan, and J. E. Sipe, "Long-period fiber gratings as band-rejection filters," J. Lightw. Technol., vol. 14, no. 1, pp. 58-64, Jan. 1996.

[2] V. Bathia and A. M. Vengsarkar, "Optical fiber long-period grating sensors," Opt. Lett., vol. 21, no. 9, pp. 692-694, 1996.

[3] S.W. James and R. P. Tatam, "Optical fibre long-period grating sensors: Characteristics and application," Meas. Sci. Technol., vol. 14, no. 5, pp. R49-R61, 2003.

[4] X. Shu, L. Zhang, and I. Bennion, "Sensitivity characteristics of longperiod fiber grating," J. Lightw. Technol., vol. 20, no. 2, pp. 255-266, Feb. 2002.

[5] I. Del Villar, I. R. Matias, F. J. Arregui, and P. Lalanne, "Optimization of sensitivity in long period fiber gratings with overlay deposition," $O p t$. Express, vol. 13, no. 1, pp. 56-69, 2005.

[6] A. Cusano et al., "Mode transition in high refractive index coated long period gratings," Opt. Express, vol. 14, no. 1, pp. 19-34, 2006.
[7] M. Smietana, M. Koba, P. Mikulic, and W. J. Bock "Measurements of reactive ion etching process effect using long-period fiber gratings," Opt. Express, vol. 22, no. 5, pp. 5988-5994, 2014.

[8] I. Del Villar, J. L. Cruz, A. B. Socorro, J. M. Corres, and I. R. Matias, "Sensitivity optimization with cladding-etched long period fiber gratings at the dispersion turning point," Opt. Express, vol. 24, no. 16, 17680$17685,2016$.

[9] I. Del Villar, "Ultrahigh-sensitivity sensors based on thin-film coated long period gratings with reduced diameter, in transition mode and near the dispersion turning point," Opt. Express, vol. 23, no. 7, pp. 8389-8398, 2015.

[10] M. Smietana, M. Koba, P. Mikulic, and W. J. Bock, "Towards refractive index sensitivity of long-period gratings at level of tens of $\mu \mathrm{m}$ per refractive index unit: fiber cladding etching and nano-coating deposition," Opt. Express, vol. 24, no. 11, pp. 11897-11904, 2016.

[11] F. Chiavaioli, C. A. J. Gouveia, P. A. S. Jorge, and F. Baldini, "Towards a uniform metrological assessment of grating-based optical fiber sensors: From refractometers to biosensors," Biosensors, vol. 7, no. 2, 2017, Art. no. E23.

[12] E. Anemogiannis, E. N. Glytsis, and T. K. Gaylord, "Transmission characteristics of long-period fiber gratings having arbitrary azimuthal/radial refractive index variation," J. Lightw. Technol., vol. 21, no. 1, pp. 218-227, Jan. 2003.

[13] I. Del Villar, I. R. Matias, and F. J. Arregui, "Influence in cladding mode distribution of overlay deposition on long-period fiber gratings," J. Opt. Soc. Amer. A., vol. 23, pp. 651-658, 2006.

[14] V. Bhatia et al., "Temperature-insensitive and strain-insensitive longperiod grating sensors for smart structures," Opt. Eng., vol. 36, no. 7 , pp. 1872-1876, Jul. 1997.

[15] T. A. Birks, P. St. J. Russell, and D. O. Culverhouse, "The acoustooptic effect in single-mode fiber tapers and couplers," J. Lightw. Technol., vol. 14, no. 11, pp. 2519-2529, Nov. 1996.

[16] I. Del Villar et al., "Sensitivity enhancement in low cutoff wavelength long-period fiber gratings by cladding diameter reduction," Sensors, vol. 17, 2017, Art. no. E2094.

[17] R. Guyard, D. Leduc, C. Lupi, and Y. Lecieux, "Global overview of the sensitivity of long period gratings to strain," Opt. Laser Technol., vol. 79 pp. $62-73,2016$.

[18] V. Bhatia, "Applications of long-period gratings to single and multiparameter sensing," Opt. Express, vol. 4, pp. 457-66, 1999.

Ignacio Del Villar received the M.S. degree in electrical and electronic engineering and the $\mathrm{Ph} . \mathrm{D}$. degree, specialty in optical fiber sensors, in 2002 and 2006, respectively, from the Public University of Navarra, Navarra, Spain. During 2004, he was a Visiting Scientist with the Institute d'Optique, Orsay, France, and in 2005 he was a Visiting Scientist with the Applied Physics Department, University of Valencia, Burjassot, Spain. He has been a Reader with the Public University of Navarra since 2008, an Associate Editor of the Optics \& Laser Technology Journal since 2012 and of Sensors Journal since 2017. His research interests include optical fiber sensors and the effect of nanostructured coatings deposited on waveguides, where he has co-authored more than 100 chapter books, journals, and conference papers.

Omar Fuentes has been an Automatic Engineer with Higher Polytechnic Institute "José A. Echeverria (CUJAE), La Habana, Cuba," since 1996. He currently works as an Assistant Professor with the Department of Telecommunication and Electronic Engineering, Pinar del Río University, Pinar del Río, Cuba. 433 
8

JOURNAL OF LIGHTWAVE TECHNOLOGY

450 Francesco Chiavaioli received the M.Eng. degree summa cum laue in telecoms451 munications engineering and the Ph.D. degree in information engineering from the University of Siena, Italy, in 2008 and 2012, respectively. He is working as a fixed-term Researcher with the Institute of Applied Physics "Nolo Carrara" (CNR-IFAC), Florence, Italy, in the design and characterization of optical fiber sensors, especially those based on fiber gratings (FBG and LPG), for the deterdesign and characterization of combined bile and $\mathrm{pH}$ fiber probes in collaboraion with University Hospital "Careggi." He focused on both the use of LPG to couple light into WGM microresonators and SPR devices. In 2015-2016, he spent six months as a Visiting Scientist with the Institute of Photonic Sciences,
Barcelona, Spain, during which he worked with graphene-based nanocavities and optical modulators. From half of 2016, he started collaborating with the University of Navarro, Pamplona, Spain, in the development and characterzation of loss mode resonance fiber devices. He is author of more than 40 publications on the subjects in ISI Journals and in Conference Proceedings. He is member of OSA, EOS, and SIOF.
Jesus M. Corries received the M.S. degree in electrical engineering and the Ph.D. degree from the Public University of Navarro, (UPNA), Pamplona, Spain, in 1996 and 2003, respectively. He currently works as an Associate Professor
with the Department of Electrical and Electronic Engineering. UPNA. His main research is the development of fiber optic sensors using nanostructured materats for biomedical, environmental and safety applications. He is the author or coauthor of more than 100 publications. He serves as an Associate Editor of IEEE SENSOR LETTERS and Hindawi Journal of Sensors.

Ignacio R. Matias (SM'03) received the M.S. degree in electrical and electronic engineering and the PhD. degree in optical fiber sensors from the Polytechnic University of Madrid, Madrid, Spain, in 1992 and 1996, respectively. He became
a Lecturer with the Public University of Novara, Pamplona, Spain, in 1996, where currently he is a Permanent Professor. He has coauthored more than 300 chapter books, journals, and conference papers related to optical fiber sensors and passive optical devices and systems. He is a Senior Editor of IEEE SENSORS
JOURNAL. He is a Topical Editor of the IEEE SENSORS JOURNAL.

468
469
470
471
472
473
474
475
476
477
478
479
480
481
482
483
484
485 


\title{
Optimized Strain Long-Period Fiber Grating (LPFG) Sensors Operating at the Dispersion Turning Point
}

\author{
Ignacio Del Villar, Omar Fuentes, Francesco Chiavaioli, Member, IEEE, Jesus M. Corres, Member, IEEE, \\ and Ignacio R. Matias, Senior Member, IEEE
}

\begin{abstract}
Two phenomena for enhancing the sensitivity of longperiod fiber gratings are combined toward an increase of the sensitivity to strain of this type of devices: the dispersion turning point (DTP) and the cladding diameter reduction by an etching process. The results prove that sensitivities up to $20 \mathrm{pm} / \mu \varepsilon$ can be attained, which is a ten-fold improvement compared to the previous works. The sensitivity in the grating region, which is subjected to etching, does not depend on the order of the cladding mode responsible for the attenuation bands generated in the transmission spectrum, but on the proximity to the DTP for each mode order. On the other hand, the sensitivity to strain of the global structure, including the region without etching, can be increased for lower order modes in a perceptible way if the length of the etched region is smaller compared to the fiber region under stress. The experimental results are supported with simulations based on coupled-mode theory and on FIMMWAVE, which allows understanding the phenomena involved during the sensing process.
\end{abstract}

Index Terms-Dispersion turning point, etching, long period fiber grating, strain sensor.

\section{INTRODUCTION}

$\mathbf{L}$ ONG period fiber gratings (LPFGs) consist typically of a periodic perturbation in the core of a single mode optical fiber, which allows a co-propagating coupling of light from the guided mode in the fiber core to several modes guided in the cladding [1]-[3]. This provides LPFGs with sensitivity to strain, temperature, bending and refractive index of the surrounding medium [3]. In order to guarantee that a co-propagating coupling to the cladding modes occurs, the period must be typically higher than $100 \mu \mathrm{m}$ (FBGs are generated with shorter periods and, hence, a counter-propagating coupling is generated).

During the last two decades, three main ways of enhancing the sensitivity of LPFGs to the surrounding refractive index (SRI)

Manuscript received November 7, 2017; revised January 2, 2018; accepted January 2, 2018. This work was supported in part by the Spanish Ministry of Economy and Competitiveness (TEC2016-78047-R - AEI/FEDER, UE), in part by the Government of Navarra PI044 NANOSEN, and in part by the Pinar del Río University. (Corresponding author: Ignacio Del Villar.)

I. Del Villar, J. M. Corres, and I. R. Matias are with the Institute of Smart Cities and the Electrical and Electronic Engineering Department, Public University of Navarre, Pamplona 31006, Spain (e-mail: ignacio.delvillar@unavarra.es; jmcorres@unavarra.es; natxo@unavarra.es).

O. Fuentes is with the Department of Telecommunications and Electronics, Pinar del Río University, Pinar del Río 20100, Cuba (e-mail: omarf@upr.edu.cu).

F. Chiavaioli is with the "Nello Carrara" Institute of Applied Physics, National Research Council of Italy, Firenze 50019, Italy (e-mail: f.chiavaioli @ ifac.cnr.it).

Color versions of one or more of the figures in this paper are available online at http://ieeexplore.ieee.org.

Digital Object Identifier 10.1109/JLT.2018.2790434 have been explored: the selection of an adequate period that allows the LPFG to operate at the dispersion turning point (DTP) [4], the deposition of a thin-film that allows the device operating in mode transition [5], [6] and the reduction of the cladding diameter [7], [8]. The combination of these three effects allows attaining sensitivities comparable or even higher than surface plasmon resonance sensors (SPRs) [9], [10], which positions LPFGs as one of the most promising platforms in the field of optical sensors.

A high sensitivity to SRI is a good indicator for the ability of the sensor to be used as a chemical or a biological sensor, because the parameter to detect (e.g., an antigen, antibody, or any chemical species) is deposited on the surface of the sensor and, consequently, affects the medium surrounding the optical fiber [11]. However, few research has been devoted to the optimization of the strain sensitivity, which not necessarily follows the same rules as the SRI sensitivity.

In this work, focus is centered on the combination of two phenomena: the DTP and the cladding etching. By combining both effects, it will be demonstrated that, contrary to what happens with the sensitivity to SRI [8], coupling to lower order cladding modes does not allow attaining a better sensitivity. However, for each cladding mode, the sensitivity is improved when the DTP is approaching.

In addition, another way of improving the sensitivity is applying stress in a longer region than the one that has been etched. The sensitivity to strain is increased for lower order modes (i.e., shorter diameter) compared to higher order modes, and this improvement is better if the length of region under stress is much longer than the region that has been etched.

In Section II the experimental setup and the methods used for fabricating and simulating the LPFGs are described. In Section III the experimental results are detailed. Finally, some conclusions are presented in Section IV.

\section{EXPERIMENTAL SECTION}

LPFGs with a grating period of $191 \mu \mathrm{m}$ and a length of $19 \mathrm{~mm}$ were written in a photosensitive boron-germanium codoped optical fiber (Fibercore PS1250/1500) having mode field diameter of $9.6 \mu \mathrm{m}$, numerical aperture of 0.13 and cladding diameter of $125 \mu \mathrm{m}$.

The LPFGs were fabricated with the point-by-point inscription technique by using an excimer $\mathrm{KrF}$ laser source (LAMBDA Physik COMPex 110, Coherent Inc.) operating at a wavelength of $248 \mathrm{~nm}$, and working at constant pulse energy. The other 


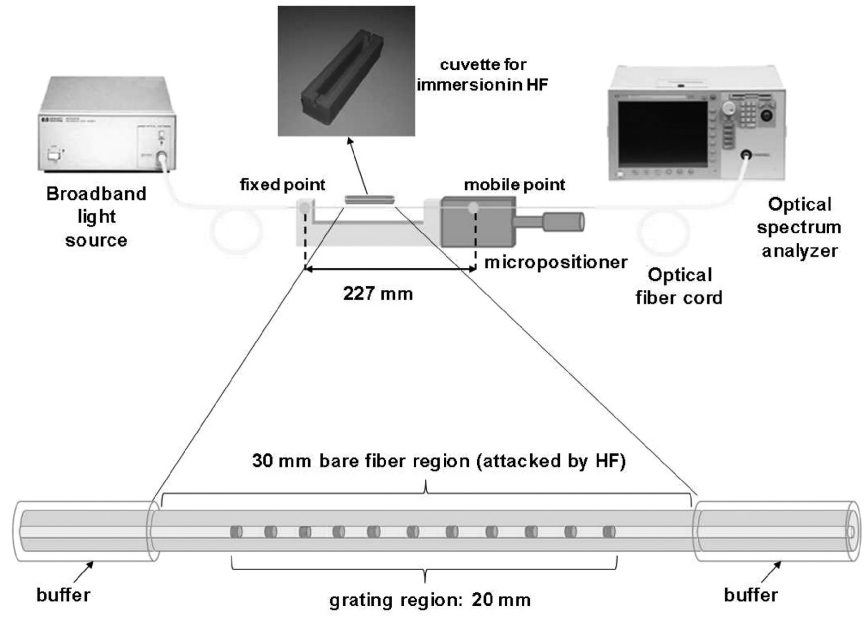

Fig. 1. Experimental setup used to etch the optical fiber containing an LPFG.

manufacturing parameters are as follows: energy of $140 \mathrm{~mJ}$ fluence of $200-300 \mathrm{~mJ} / \mathrm{cm}^{-2}$, and a repetition rate of $50 \mathrm{~Hz}$.

The laser beam was focused by using a cylindrical lens before being passed through a micrometric slit placed in front of the optical fiber, which determined the grating period. The fiber was kept straight during both the fabrication and the characterization processes to avoid any bending artifact.

The LPFGs were subjected to an etching process depicted in Fig. 1. The process was monitored with a transmission configuration setup. Light from an Agilent 83437A broadband source was launched into the LPFG during the etching process and the output light was monitored in an Agilent 86140B optical spectrum analyzer, which allowed observing the evolution of the attenuation bands and hence stopping at the adequate position.

Regarding the etching process, the fiber was introduced in a plastic cuvette filled with $40 \%$ hydrofluoric acid. The cuvette contains two 0.9-mm-wide grooves where the fiber segment to etch can be placed without affecting the rest of the fiber and without releasing acid out of the cuvette. The length of the fiber attacked by the acid was $30 \mathrm{~mm}$, enough to include the $19 \mathrm{~mm}$ long grating portion. In order to guarantee an adequate etching, the fiber was also fixed to an outer plastic holder and to a mobile point in a micropositioner, which ensured that the fiber was kept straight, avoiding bend-induced distortion of the transmission spectrum. The region not immersed in the cuvette was $197 \mathrm{~mm}$ long, for a global length of the fiber under stress of $227 \mathrm{~mm}$.

When the attenuation bands were positioned at a specific wavelength, the fiber was extracted from the cuvette and washed using water immediately after to eliminate the effects of acid.

The process can be repeated as many times as necessary in order to further reduce the cladding diameter, which allows obtaining attenuation bands corresponding with other cladding modes [8]. Moreover, if it is necessary to control the position of the attenuation bands with more accuracy, the LPFG can also be immersed in a more diluted HF solution.

After the etching process, it was essential to wait at least one hour to ensure that the LPFG was completely dry. Once dried, the LPFG was ready for performing strain measurements. To this purpose, as indicated in Fig. 1, the LPFG was manually stretched in steps of $0.1 \mathrm{~mm}$ (the transmission spectrum of the sensor was continuously monitored during the stretching process).

It is important to highlight that the diameter of the fiber was just reduced in the region of the LPFG immersed in the cuvette. Consequently, the strain in the grating can be obtained according to this expression:

$$
\varepsilon_{1}=\frac{\frac{\Delta L}{A_{1}}}{\left(\frac{L_{1}}{A_{1}}+\frac{L_{2}}{A_{2}}\right)}
$$

where $\Delta \mathrm{L}$ is the overall length increase of the LPFG region under stress, $L_{1}$ and $L_{2}$ are the lengths of the etched and nonetched fiber portions respectively, $A_{1}$ is the cross section of the etched fiber portion and $\mathrm{A}_{2}$ is the cross section of the non-etched fiber portion.

\section{THEORY}

The structures studied in this work were analyzed with two software tools: FIMMWAVE and a method based on coupledmode theory [13]. FIMMWAVE, which is a fast and efficient software for analyzing optical waveguides, was used for generating the transmission spectra, whereas the coupled-mode theory was used for obtaining the resonance wavelengths that meet the phase-matching condition (this last operation is less computationally demanding and cannot be directly obtained with FIMMWAVE).

Regarding FIMMWAVE, the propagation was calculated with FIMMPROP, a module integrated with FIMMWAVE. For LPFG sections, the finite difference method (FDM) was used, because it is the most accurate method available for a cylindrical waveguide.

The grating used in the simulations consisted of a square wave that emulates the point-by-point technique used during the inscription of the grating. The peak-to-peak modulation was $8 \times$ $10^{-4}$. Modes $\mathrm{LP}_{0,1}$ up to $\mathrm{LP}_{0,12}$ were analyzed for the period of the LPFGs used experimentally. $\mathrm{LP}_{0,11}$ was the higher-order cladding mode to which the core mode is coupled when the cladding diameter is $125 \mu \mathrm{m}$, i.e., before the fiber was etched.

By fitting the experimental results presented in Section IV with the theoretical ones, a core diameter of $6.9 \mu \mathrm{m}$ and a numerical aperture of 0.1313 at wavelength of $1300 \mathrm{~nm}$ was calculated, very close to 0.13 numerical aperture given by the manufacturer. For a better accuracy, a negative dispersion of $1.1 \times 10^{-7}(\lambda-1300)^{2}$ was added, where $\lambda$ is the operating wavelength.

It is well known that the resonance wavelength of an LPFG is determined by the phase-matching condition [1]-[4]:

$$
\lambda=\left[n_{\text {core }}(\lambda)-n_{\text {clad }}^{i}(\lambda)\right] \Lambda
$$

where $n_{\text {core }}(\lambda)$ is the effective refractive index of the propagating core mode at wavelength $\lambda, n_{\text {clad }}^{i}(\lambda)$ is effective the refractive index of the $i$ th cladding mode and $\Lambda$ is the period of the grating.

However, the modified phase-matching condition is [12]:

$$
\lambda=\left[n_{\text {core }}(\lambda)+\frac{s_{0}}{k_{0}} \varsigma_{\text {core }}-\left(n_{\text {clad }}^{i}(\lambda)+\frac{s_{0}}{k_{0}} \varsigma_{\text {clad }}^{i}\right)\right] \Lambda
$$


where the additional variables $s_{0}, k_{0}, \zeta_{\text {core }}$ and $\zeta_{\text {clad }}^{i}$ are the first Fourier component of the grating profile, the free space wavenumber, the self-coupling coefficient of the core mode and the self-coupling coefficient of the cladding mode, respectively, allows improving the accuracy of the equation towards a prediction of the position of the attenuation bands in the transmission spectrum [12]. Consequently, (3) will be used for the analysis performed in this section according to coupled-mode theory [13].

Using the chain rule of derivatives, the sensitivity to axial strain $\varepsilon$ can obtained from (3) as follows:

$$
\frac{d \lambda}{d \varepsilon}=\frac{d \lambda}{d m a t} \frac{d m a t}{d \varepsilon}+\Lambda \frac{d \lambda}{d \Lambda}
$$

where the first element on the right side of the equation is related to the material contribution extracted from expression (3):

$$
\text { mat }=\left[n_{\text {core }}(\lambda)+\frac{s_{0}}{k_{0}} \zeta_{\text {core }}-\left(n_{\text {clad }}^{i}(\lambda)+\frac{s_{0}}{k_{0}} \varsigma_{\text {clad }}^{i}\right)\right]
$$

This contribution results from the elasto-optic effect (i.e., the change in refractive index in both the fiber core and the cladding due to strain) and the Poisson's effect (i.e., the change in the transverse dimensions). The second element in expression (4) is the waveguide contribution, which depends on the slope $\mathrm{d} \lambda / \mathrm{d} \Lambda$ of the characteristic curve of the resonance band [3], [14].

The elasto-optic coefficient of silica is a well know parameter, -0.22 [4], [15], whereas the elasto-optic coefficient of the fiber core is not given in the literature because it depends on the doping level of the materials used by the manufacturer. The value that best fitted the experimental results in Section IV was -0.222 , which is in the range of the values explored in [4].

In order to analyze the effect of both the material and waveguide contributions in the optical fiber used in this work, two situations were taken into account: an LPFG without etching and an etched LPFG.

Fig. 2(a) shows the dependence of the coupling wavelength for cladding modes $\mathrm{LP}_{0,2}-\mathrm{LP}_{0,12}$ upon the period of the LPFGs used. For $\mathrm{LP}_{0,11}$ the DTP was observable [4]. This phenomenon is related to a high sensitivity. Consequently, the best option was to choose a period of $191 \mu \mathrm{m}$, which cuts the curve of $\mathrm{LP}_{0,11}$ cladding mode at two points very close to the DTP, where the sensitivity is very high.

It was also proved experimentally that, by reducing the cladding diameter of the optical fiber, coupling to lower order cladding modes in the DTP was possible [8]. This idea is confirmed in Fig. 2(b), where for an LPFG of cladding diameter $65.45 \mu \mathrm{m}$ a coupling to $\mathrm{LP}_{0,6}$ cladding mode at DTP is obtained.

In Fig. 3 the waveguide and the material contribution were analyzed with a focus on grating periods close to $191 \mu \mathrm{m}$, the period selected for the LPFGs analyzed in Section IV. To this purpose, the coupling wavelength for cladding mode $\mathrm{LP}_{0,11}$ was calculated in two conditions: one where the refractive index of both the fiber core and cladding was that corresponding to no strain applied to the LPFG, and another where the refractive index of both the fiber core and cladding was that corresponding to $3000 \mu \varepsilon$ applied to the LPFG. The effect is more evident near the DTP. However, it is very small if compared with the

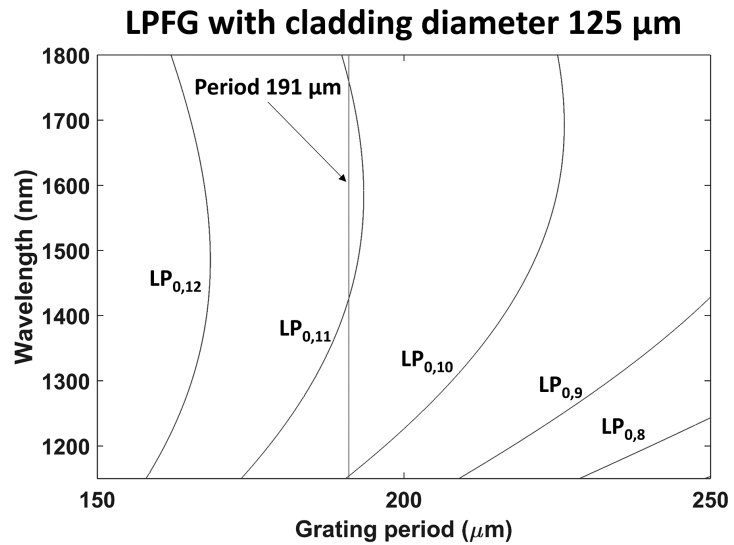

(a)

LPFG with cladding diameter $65.45 \mu \mathrm{m}$

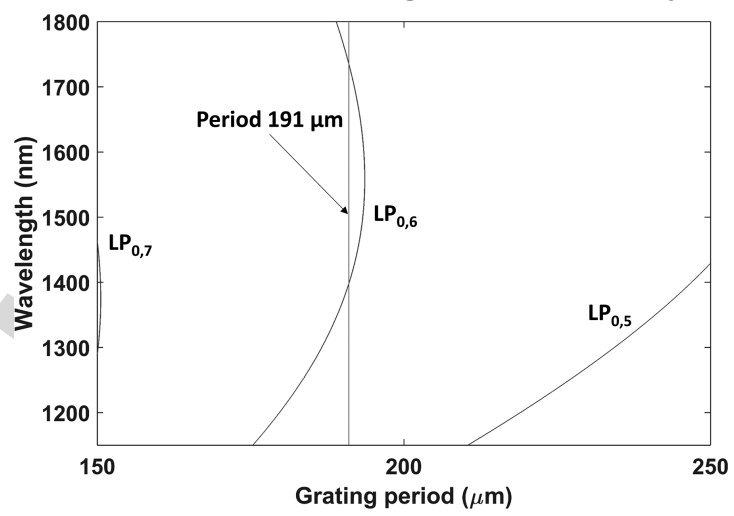

(b)

Fig. 2. Calculated variation of resonance wavelength with grating period: (a) For a cladding diameter of $125 \mu \mathrm{m}$ (for a period of $191 \mu \mathrm{m}$, the $\mathrm{LP}_{0,11}$ phase matching curve is intersected at two wavelengths very close to the dispersion turning point); (b) For a cladding diameter of $65.45 \mu \mathrm{m}$ (for a period of $191 \mu \mathrm{m}$, the $\mathrm{LP}_{0,6}$ phase matching curve is intersected at two wavelengths very close to the dispersion turning point).

effect of increasing the grating period by $0.3 \%$, equivalent to $3000 \mu \varepsilon$ in the LPFG (see the vertical lines of $191 \mu \mathrm{m}, 0 \mu \varepsilon$, and $191.576 \mu \mathrm{m}, 3000 \mu \varepsilon$ ). In Fig. 3(a), for an LPFG with cladding diameter $125 \mu \mathrm{m}$, a wavelength shift of approximately $10 \mathrm{~nm}$ was observed (waveguide contribution) compared to less than $1 \mathrm{~nm}$ induced by the material contribution. In other words, the effect of the waveguide is predominant over the effect of the material. The same effect was observed for an LPFG with diameter $65.45 \mu \mathrm{m}$ in Fig. 3(b).

Another interesting conclusion that was extracted from the results obtained with two different diameters and shown in Fig. 3 was that the shape of the phase matching curve did not change. This indicates that the optimized sensitivity to strain is achieved for any mode as long as it operates at or close to the DTP point Consequently, the basic rule for optimizing the sensitivity to strain should be to approach the DTP point. In addition to this, it is easy to observe in Fig. 3 the non-linear wavelength shift of the coupling wavelength as a function of the grating period in the proximities of the DTP.

\section{EXPERIMENTAL RESULTS}

According to Sections II and III, different cladding diameters were explored (see Table I). The diameter was estimated by 
LPFG with cladding diameter $125 \mu \mathrm{m}$

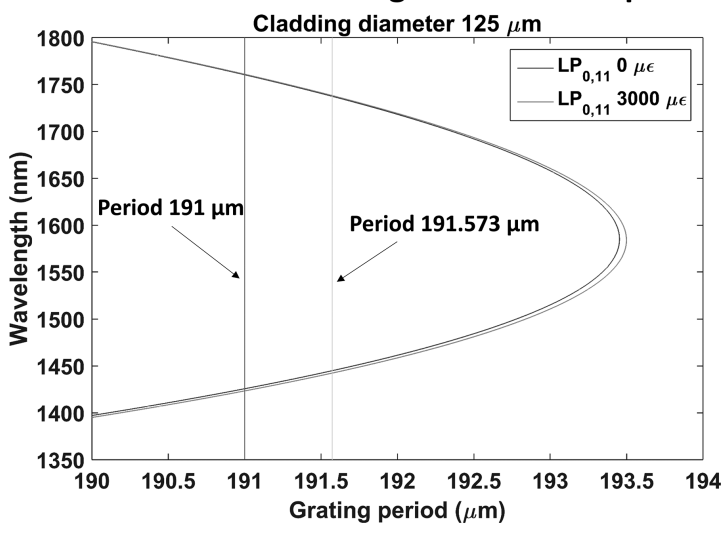

(a)

LPFG with cladding diameter $65.45 \mu \mathrm{m}$

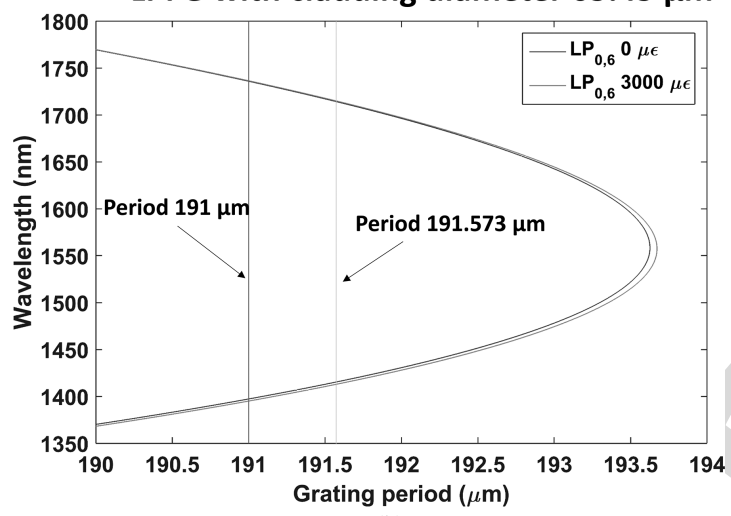

(b)

Fig. 3. Effect of the material in the variation of resonance wavelength with grating period ( 0 and $3000 \mu \varepsilon$ are compared), and effect of the waveguide (a period of $191 \mu \mathrm{m}$ is compared with $191.576 \mu \mathrm{m}$, which is a $0.3 \%$ more to represent the effect of $3000 \mu \varepsilon$ ): (a) For a cladding diameter of $125 \mu \mathrm{m}$. (b) For a cladding diameter of $65.45 \mu \mathrm{m}$.

TABLE I

LIST OF LPFG SENSORS OF PERIOD $191 \mu \mathrm{M}$

\begin{tabular}{llll}
\hline Sensor & Mode order & Lambda $(\mathrm{nm})$ & \multicolumn{1}{c}{ Diameter $(\mu \mathrm{m})$} \\
\hline S0 & $\mathrm{LP}_{0,11}$ & 1461 & 125 \\
S1 & $\mathrm{LP}_{0,11}$ & 1489 & 124.68 \\
S2 & $\mathrm{LP}_{0,11}$ & 1508 & 124.56 \\
S3 & $\mathrm{LP}_{0,10}$ & 1317 & 115.82 \\
S4 & $\mathrm{LP}_{0,10}$ & 1393 & 113.98 \\
S5 & $\mathrm{LP}_{0,10}$ & 1456 & 113.10 \\
S6 & $\mathrm{LP}_{0,9}$ & 1392 & 101.94 \\
S7 & $\mathrm{LP}_{0,9}$ & 1455 & 101.16 \\
S8 & $\mathrm{LP}_{0,8}$ & 1390 & 89.90 \\
S9 & $\mathrm{LP}_{0,6}$ & 1462 & 65.31 \\
\hline
\end{tabular}

relating the position of the attenuation bands, after each etching process, with the same position obtained in the theoretical transmission spectra. It is important to note that a good correspondence between the estimation of the diameter and the experimental value of the diameter measured in a microscope was demonstrated in [16].

S0 was an LPFG without etching (diameter $125 \mu \mathrm{m}$ ). The device was subjected to stress according to the method explained in Section II. In Fig. 4(a), the separation between the two

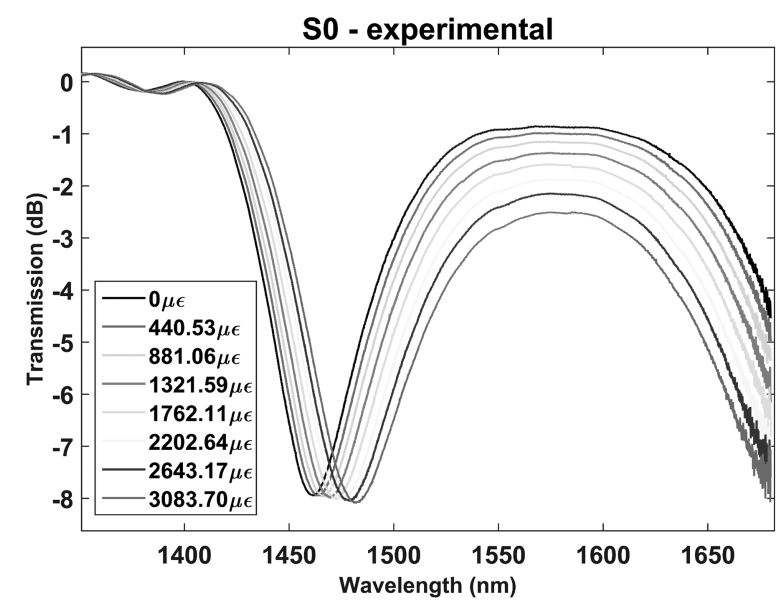

(a)

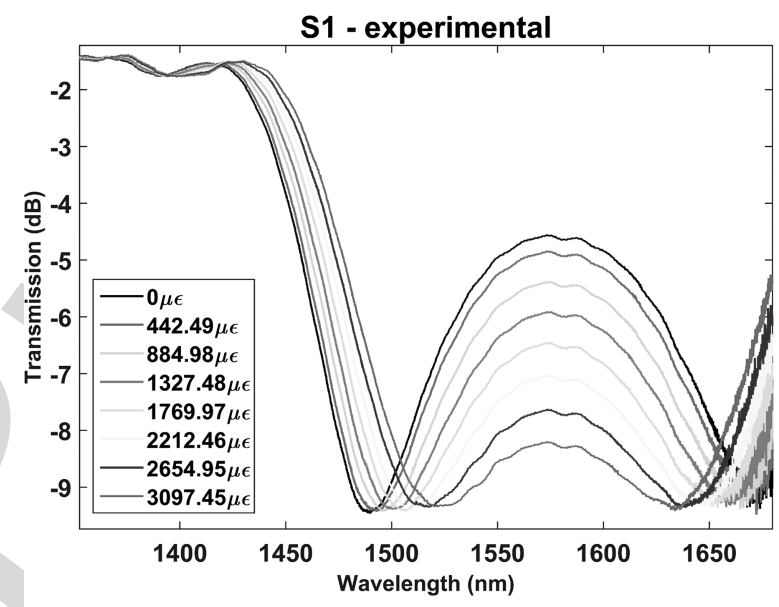

(b)

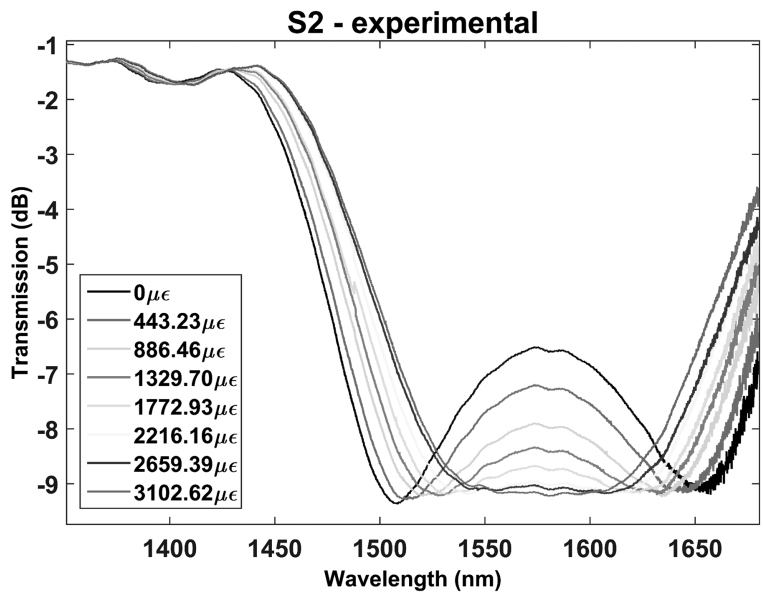

(c)

Fig. 4. Transmission spectra (experimental results) for: (a) S0; (b) S1; (c) S2.

attenuation bands observed in the optical spectrum is reduced 250 as a function of strain. This agrees with Fig. 3(a), where an 251 increase in the grating period leads to an approach to the DTP. 252

By performing a soft etching, we fabricated S1 with an esti- 253 mated diameter of $124.68 \mu \mathrm{m}$, according to the numerical results 254 presented in Fig. 5. Following the analysis in [8], [9], the sep- 255 aration of the attenuation bands decreases if the diameter of 256 an LPFG is reduced. This is what was observed in Fig. 4(b) if 257 


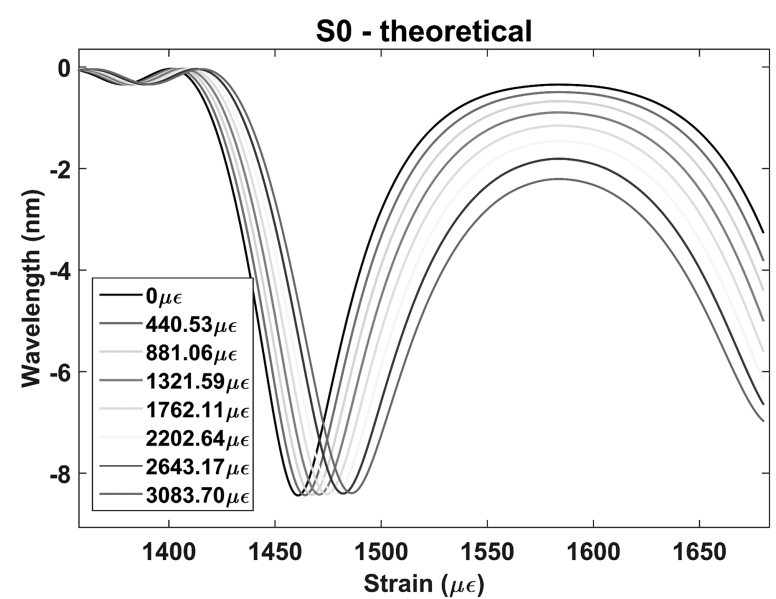

(a)

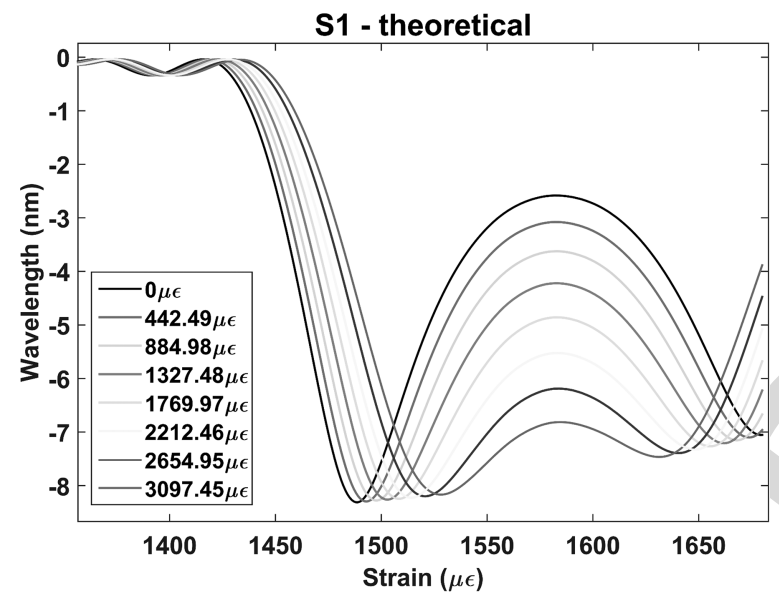

(b)

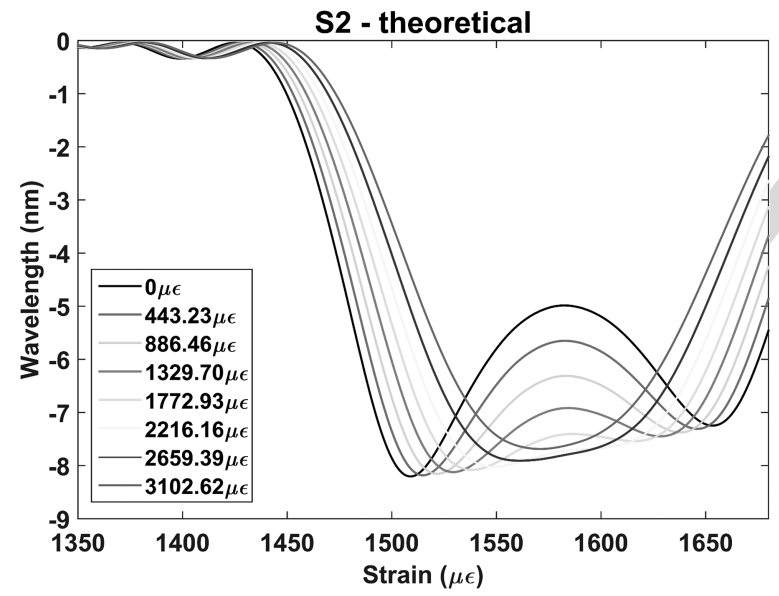

(c)

Fig. 5. Transmission spectra (theoretical results) for: (a) S0; (b) S1; (c) S2.

compared with Fig. 4(a). The same occurred for sensor S2 in Fig. 4(c), with an estimated diameter of $124.56 \mu \mathrm{m}$. The theoretical results in Fig. 5 confirmed the experimental results of Fig. 4.

In addition, the central wavelength of the left band is plotted in Fig. 6 as a function of strain for all sensors analyzed in Figs. 4 and 5. The theoretical and experimental results allowed obtaining several conclusions. The sensitivity increases

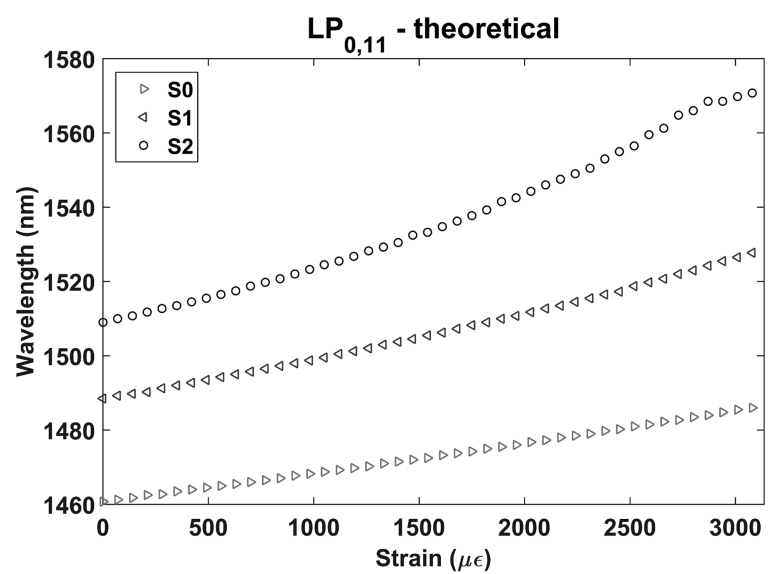

(a)

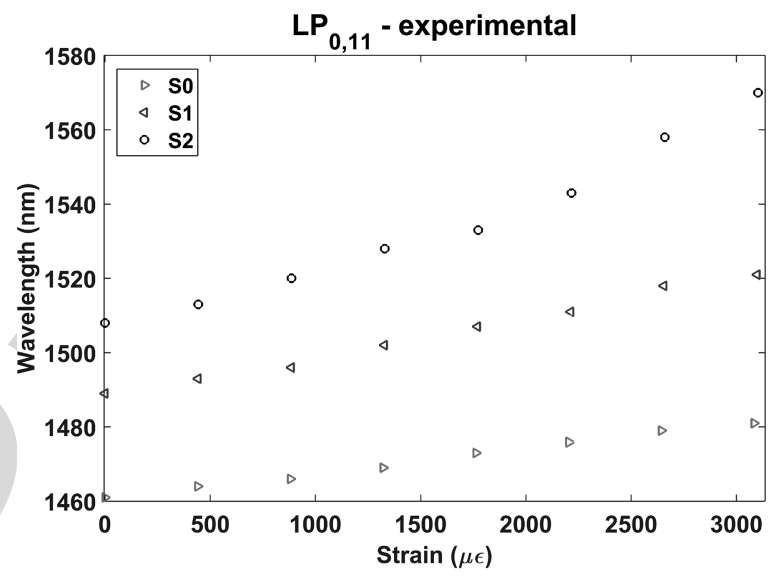

(b)

Fig. 6. Wavelength shift of $\mathrm{LP}_{0,11}$ left band as a function of strain for sensors S0, S1 and S2: (a) theoretical results; (b) experimental results.

if the diameter is reduced (the sensitivity of S0, S1 and S2 is 266 $6.5 \mathrm{pm} / \mu \varepsilon, 10.3 \mathrm{pm} / \mu \varepsilon$ and $20 \mathrm{pm} / \mu \varepsilon$, respectively, in the range 267 0-3100 $\mu \varepsilon)$. For the sake of comparison, the sensitivity in op- 268 timized LPFGs ranged from 0.5 to $2 \mathrm{pm} / \mu \varepsilon$ in [17], whereas 269 in [18] the maximum sensitivity was $2 \mathrm{pm} / \mu \varepsilon$. This indicates 270 that our best sensor improved the highest sensitivity attained in 271 these works by one order of magnitude.

A second conclusion is that if the separation between the attenuation bands is low, as S2 in Fig. 5, the relation between wavelength and strain is non-linear, whereas this relation is linear for sensor S0. Sensor S1 is in the middle between both situations. This agrees with what was observed in Fig. 3, where the phase matching curve is non-linear in the proximities of the DTP.

A harder etching was performed up to a diameter that allowed monitoring attenuation bands that were due to coupling to $\mathrm{LP}_{0,10}$ cladding mode. Three different diameters were analyzed: $115.82,113.98$ and $113.10 \mu \mathrm{m}$ (sensors S3, S4 and S5).

Theoretical and experimental data are presented in Fig. 7 for the central wavelength of the left band versus different values of strain. In all cases, the relation between strain and wavelength was linear. This can be explained because this time focused was centered on sensors working far from DTP: sensor S5 was working at the same wavelength as S0 in Fig. 6, where a 70 271
272 273 274 275 276 277 278 279 280 281 282 283 284 285 286 287 288 289 


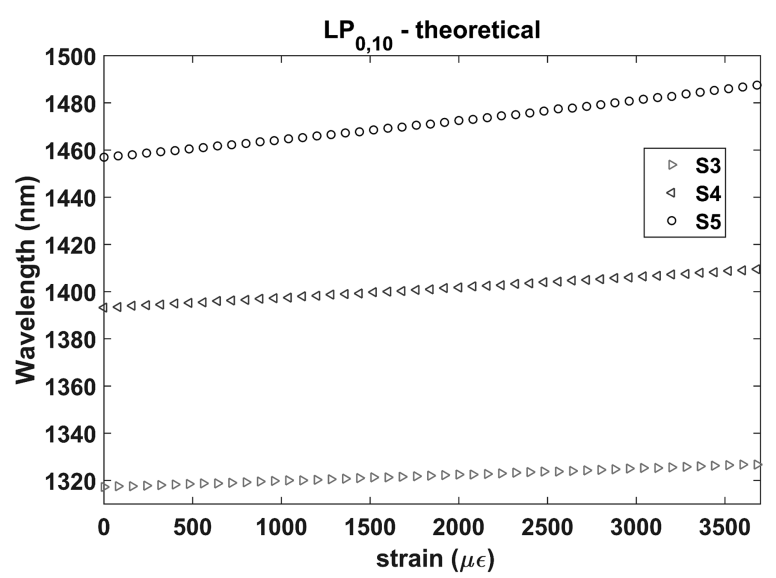

(a)

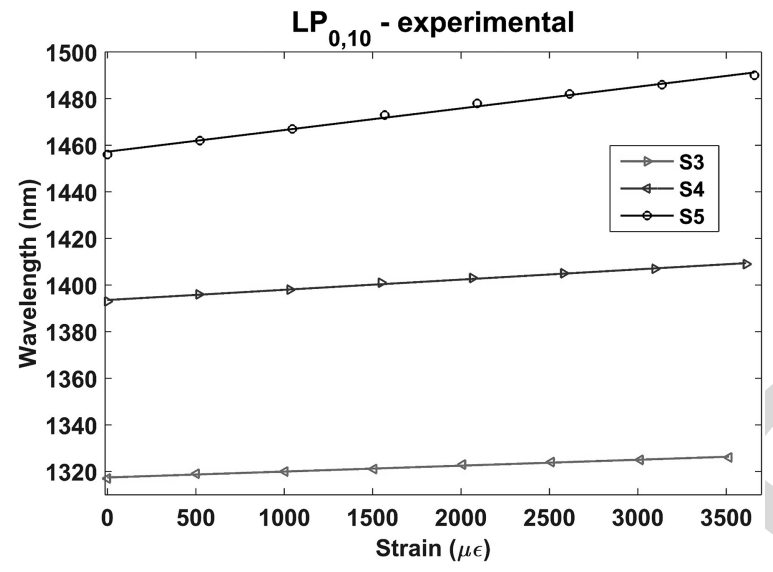

(b)

Fig. 7. Wavelength shift of $L P_{0,10}$ left band as a function of strain for sensors S3, S4 and S5: (a) theoretical results; (b) experimental results. linear performance was observed, and S3 and S4 were working at shorter wavelengths. In view of the linear shape of the plots, a Matlab linear regression model has been created (solid lines in Fig. 7) that fits with the experimental points. This model also allows obtaining the root-mean-square deviation along with the sensitivities for $\mathrm{S} 3, \mathrm{~S} 4$ and $\mathrm{S} 5$. The sensitivities are $2.5 \mathrm{pm} / \mu \varepsilon$, $4.4 \mathrm{pm} / \mu \varepsilon$ and $9.3 \mathrm{pm} / \mu \varepsilon$, respectively, in the range $0-3500 \mu \varepsilon$, whereas the root-mean-square deviations (RMSD) are respectively $0.348 \mathrm{~nm}, 0.432 \mathrm{~nm}$ and $1.05 \mathrm{~nm}$. The highest RMSD is obtained for S5, the sensor with the highest sensitivity and the sensor closer to DTP, where a non-linear dependence with strain is observed. In addition, as shown in Fig. 6, the sensitivity increases as the diameter is reduced.

In order to obtain more information on the influence of the mode order, other sensors with different diameters (S6, S7, S8 and S9) were analyzed. In Fig. 8, the performance of all sensors was compared. We divided them into two groups. Fig. 8(a) details the sensors working at a wavelength close to DTP. It seems that the sensitivity is similar. However, it is difficult to extract a general rule because the high sensitivity at this point is responsible for variations in the sensitivity of each sensor depending on small changes in the wavelength where it operates. On the other hand, Fig. 8(b) accounts for sensors operating far from the DTP, where the devices are not so sensitive to

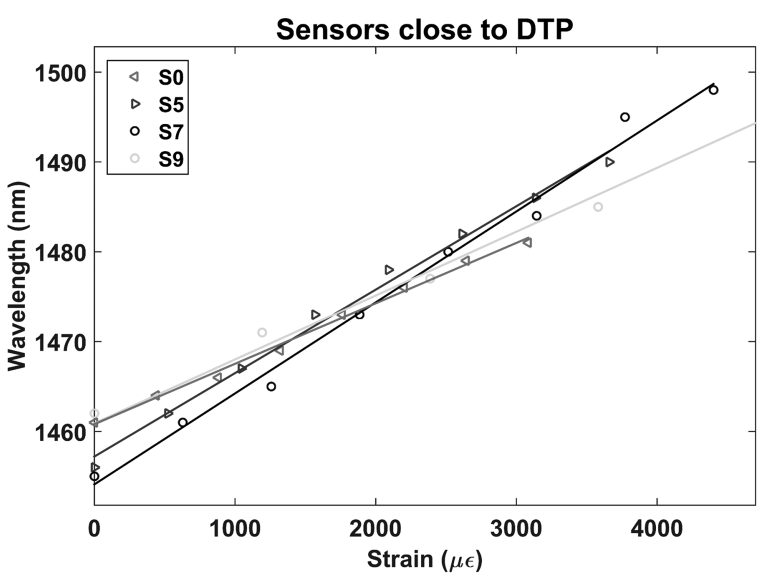

(a)

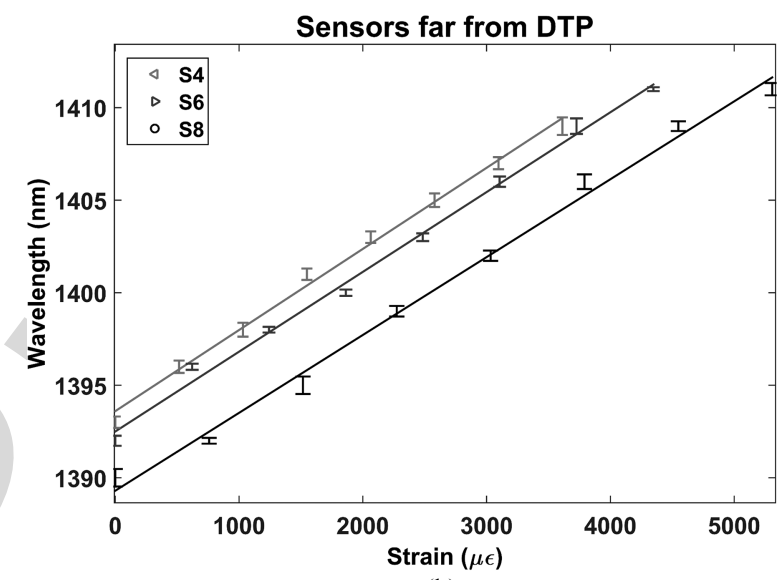

(b)

Fig. 8. Performance of LPFG sensors with different diameter: (a) S0, S5, S7 and S9 are close to DTP and the attenuation band is due to coupling to $\mathrm{LP}_{0,11}, \mathrm{LP}_{0,10}, \mathrm{LP}_{0,9}$ and $\mathrm{LP}_{0,6}$ respectively; (b) S4, S6 and $\mathrm{S} 8$ are far from DTP and the attenuation band is due to coupling to $\mathrm{LP}_{0,10}, \mathrm{LP}_{0,9}$ and $\mathrm{LP}_{0,8}$ respectively.

small variations in the operating wavelength and it is easier to 314 conclude that their sensitivity is quite similar. This indicates that 315 the mode order played no role on the sensitivity to strain of the 316 device. In other words, for each specific mode, the proximity to 317 the DTP determines the sensitivity of the device, whereas the 318 mode order has no influence on that. In this way, sensors can 319 be classified into two groups: operating at and far from DTP 320 point. Consequently, a soft etching to position the attenuation 321 bands in the optical spectrum is the best way for controlling the 322 sensitivity of the LPFG-based strain sensor.

It must be pointed out that the results presented in Figs. 4-8 324 represent the strain in the grating region of the LPFG. How- 325 ever, if the deformation in all the fiber structure is analyzed (see 326 Fig. 1), the sensitivity of the device is better for lower order 327 modes (i.e., lower diameter). Moreover, depending on the ra- 328 tio between the grating length and the complete LPFG region 329 length under stress, the sensitivity can be further improved. In 330 this sense, this ratio should be as low as possible. In the cases 331 analyzed in this work, if the strain on the global structure is con- 332 sidered, each $0.1 \mathrm{~mm}$ deformation in a structure of $227 \mathrm{~mm}$ is 333 $0.44 \mu \varepsilon$. Consequently, the $4 \mathrm{pm} / \mu \varepsilon$ sensitivity of S4, S6 and S8 334 in Fig. 8(b) becomes $5.19 \mathrm{pm} / \mu \varepsilon, 6.17 \mathrm{pm} / \mu \varepsilon$ and $6.82 \mathrm{pm} / \mu \varepsilon, \quad 335$ 
respectively, for the same sensors. These values are calculated by dividing the wavelength shift by $0.44 \mu \varepsilon$ multiplied by the seven $0.1 \mathrm{~mm}$ deformation steps analyzed in Fig. 8(b).

The RMSD of S4, S6 and S8 was also calculated: 0.432, 0.506 and $0.642 \mathrm{~nm}$ respectively. These values indicate no significant changes because the three sensors are positioned at a similar distance of the DTP. In addition, error bars representing the standard deviation in each point have been added for S4, S6 and S8 (the average standard deviation of the points in S4, S6 and S8 was $0.351,0.347$ and $0.332 \mathrm{~nm}$ respectively).

\section{CONCLUSION}

The sensitivity to strain of long period fiber gratings (LPFGs) operating close to the dispersion turning point (DTP) has been analyzed as a function of the cladding diameter.

The results obtained indicate that by accurately approaching the DTP it is possible to increase the sensitivity to strain of the device. Therefore, the sensitivity of previous works has been improved by a factor of 10 , attaining a sensitivity of $20 \mathrm{pm} / \mu \varepsilon$ in the best case.

On the other hand, unlike for LPFG-based refractometers, reducing the fiber diameter towards coupling to lower order cladding mode does not allow increasing the sensitivity of the etched region of the fiber. This indicates that a soft etching of the initial structure without etching towards a highest sensitivity is the best way of improving the performance of the device. However, if the etched region is small compared to the global region under stress, it is possible to improve the sensitivity of the global structure for lower order cladding modes, which opens the path towards the design of different combinations of length for both the etched and the non-etched regions. In this sense, the fabrication of short gratings is crucial towards the possibility to develop different designs.

The other well-known phenomenon that could be combined with the dispersion turning point and the cladding diameter reduction is the mode transition, which according to [9], [10] allows increasing the sensitivity to the surrounding refractive index changes of LPFGs exponentially. However, this requires the nanodeposition of a thin-film and, consequently, the number of variables to take into account increases in a great manner, which is beyond the scope of this work.

\section{REFERENCES}

[1] A. M. Vengsarkar, P. J. Lemaire, J. B. Judkins, V. Bhatia, T. Erdogan, and J. E. Sipe, "Long-period fiber gratings as band-rejection filters," J. Lightw. Technol., vol. 14, no. 1, pp. 58-64, Jan. 1996.

[2] V. Bathia and A. M. Vengsarkar, "Optical fiber long-period grating sensors," Opt. Lett., vol. 21, no. 9, pp. 692-694, 1996.

[3] S.W. James and R. P. Tatam, "Optical fibre long-period grating sensors: Characteristics and application," Meas. Sci. Technol., vol. 14, no. 5, pp. R49-R61, 2003.

[4] X. Shu, L. Zhang, and I. Bennion, "Sensitivity characteristics of longperiod fiber grating," J. Lightw. Technol., vol. 20, no. 2, pp. 255-266, Feb. 2002.

[5] I. Del Villar, I. R. Matias, F. J. Arregui, and P. Lalanne, "Optimization of sensitivity in long period fiber gratings with overlay deposition," Opt. Express, vol. 13, no. 1, pp. 56-69, 2005.

[6] A. Cusano et al., "Mode transition in high refractive index coated long period gratings," Opt. Express, vol. 14, no. 1, pp. 19-34, 2006.
[7] M. Smietana, M. Koba, P. Mikulic, and W. J. Bock "Measurements of reactive ion etching process effect using long-period fiber gratings," Opt Express, vol. 22, no. 5, pp. 5988-5994, 2014.

[8] I. Del Villar, J. L. Cruz, A. B. Socorro, J. M. Corres, and I. R. Matias, "Sensitivity optimization with cladding-etched long period fiber gratings at the dispersion turning point," Opt. Express, vol. 24, no. 16, 17680$17685,2016$.

[9] I. Del Villar, "Ultrahigh-sensitivity sensors based on thin-film coated long period gratings with reduced diameter, in transition mode and near the dispersion turning point," Opt. Express, vol. 23, no. 7, pp. 8389-8398, 2015.

[10] M. Smietana, M. Koba, P. Mikulic, and W. J. Bock, "Towards refractive index sensitivity of long-period gratings at level of tens of $\mu \mathrm{m}$ per refractive index unit: fiber cladding etching and nano-coating deposition," Opt. Express, vol. 24, no. 11, pp. 11897-11904, 2016.

[11] F. Chiavaioli, C. A. J. Gouveia, P. A. S. Jorge, and F. Baldini, "Towards a uniform metrological assessment of grating-based optical fiber sensors: From refractometers to biosensors," Biosensors, vol. 7, no. 2, 2017, Art no. E23.

[12] E. Anemogiannis, E. N. Glytsis, and T. K. Gaylord, "Transmission characteristics of long-period fiber gratings having arbitrary azimuthal/radial refractive index variation," J. Lightw. Technol., vol. 21, no. 1, pp. 218-227, Jan. 2003.

[13] I. Del Villar, I. R. Matias, and F. J. Arregui, "Influence in cladding mode distribution of overlay deposition on long-period fiber gratings," J. Opt. Soc. Amer. A., vol. 23, pp. 651-658, 2006.

[14] V. Bhatia et al., "Temperature-insensitive and strain-insensitive longperiod grating sensors for smart structures," Opt. Eng., vol. 36, no. 7 pp. 1872-1876, Jul. 1997.

[15] T. A. Birks, P. St. J. Russell, and D. O. Culverhouse, "The acoustooptic effect in single-mode fiber tapers and couplers," J. Lightw. Technol., vol. 14, no. 11, pp. 2519-2529, Nov. 1996.

[16] I. Del Villar et al., "Sensitivity enhancement in low cutoff wavelength long-period fiber gratings by cladding diameter reduction," Sensors, vol. 17, 2017, Art. no. E2094.

[17] R. Guyard, D. Leduc, C. Lupi, and Y. Lecieux, "Global overview of the sensitivity of long period gratings to strain," Opt. Laser Technol., vol. 79 , pp. $62-73,2016$.

[18] V. Bhatia, "Applications of long-period gratings to single and multiparameter sensing," Opt. Express, vol. 4, pp. 457-66, 1999.

Ignacio Del Villar received the M.S. degree in electrical and electronic engineering and the $\mathrm{Ph} . \mathrm{D}$. degree, specialty in optical fiber sensors, in 2002 and 2006, respectively, from the Public University of Navarra, Navarra, Spain. During 2004, he was a Visiting Scientist with the Institute d'Optique, Orsay, France, and in 2005 he was a Visiting Scientist with the Applied Physics Department, University of Valencia, Burjassot, Spain. He has been a Reader with the Public University of Navarra since 2008, an Associate Editor of the Optics \& Laser Technology Journal since 2012 and of Sensors Journal since 2017. His research interests include optical fiber sensors and the effect of nanostructured coatings deposited on waveguides, where he has co-authored more than 100 chapter books, journals, and conference papers.

Omar Fuentes has been an Automatic Engineer with Higher Polytechnic Institute "José A. Echeverria (CUJAE), La Habana, Cuba," since 1996. He currently works as an Assistant Professor with the Department of Telecommunication and Electronic Engineering, Pinar del Río University, Pinar del Río, Cuba. 433 
8

JOURNAL OF LIGHTWAVE TECHNOLOGY

450 Francesco Chiavaioli received the M.Eng. degree summa cum laue in telecoms451 munications engineering and the $\mathrm{Ph} . \mathrm{D}$. degree in information engineering from the University of Siena, Italy, in 2008 and 2012, respectively. He is working as a fixed-term Researcher with the Institute of Applied Physics "Nolo Carrara" (CNR-IFAC), Florence, Italy, in the design and characterization of optical fiber sensors, especially those based on fiber gratings (FBG and LPG), for the detectdesign and characterization of combined bile and $\mathrm{pH}$ fiber probes in collaboraion with University Hospital "Careggi." He focused on both the use of LPG to couple light into WGM microresonators and SPR devices. In 2015-2016, he spent six months as a Visiting Scientist with the Institute of Photonic Sciences,
Barcelona, Spain, during which he worked with graphene-based nanocavities and optical modulators. From half of 2016, he started collaborating with the University of Navarro, Pamplona, Spain, in the development and characterzation of loss mode resonance fiber devices. He is author of more than 40 publications on the subjects in ISI Journals and in Conference Proceedings. He is member of OSA, EOS, and SIOF.
Jesus M. Corries received the M.S. degree in electrical engineering and the Ph.D. degree from the Public University of Navarro, (UPNA), Pamplona, Spain, in 1996 and 2003, respectively. He currently works as an Associate Professor with the Department of Electrical and Electronic Engineering, UPNA. His main research is the development of fiber optic sensors using nanostructured materald for biomedical, environmental and safety applications. He is the author or
coauthor of more than 100 publications. He serves as an Associate Editor of IEEE SENSOR LETTERS and Hindawi Journal of Sensors.

Ignacio R. Matias (SM'03) received the M.S. degree in electrical and electronic engineering and the Ph.D. degree in optical fiber sensors from the Polytechnic University of Madrid, Madrid, Spain, in 1992 and 1996, respectively. He became
a Lecturer with the Public University of Novara, Pamplona, Spain, in 1996, where currently he is a Permanent Professor. He has coauthored more than 300 chapter books, journals, and conference papers related to optical fiber sensors and passive optical devices and systems. He is a Senior Editor of IEE
JOURNAL. He is a Topical Editor of the IEEE SENSORS JOURNAL.

468
469
470
471
472
473
474
475
476
477
478
479
480
481
482
483
484
485 